\title{
Symbolic Object Code Analysis ${ }^{\star}$
}

\author{
Jan Tobias Mühlberg ${ }^{1}$, Gerald Lüttgen ${ }^{2}$ \\ 1 IBBT-DistriNet, KU Leuven, Celestijnenlaan 200A, 3001 Leuven, Belgium \\ e-mail: jantobias.muehlberg@cs. kuleuven.be \\ 2 Software Technologies Research Group, University of Bamberg, 96045 Bamberg, Germany \\ e-mail: gerald.luettgen@swt-bamberg.de
}

The date of receipt and acceptance will be inserted by the editor

\begin{abstract}
Software model checkers quickly reach their limits when being applied to verifying pointer safety properties in source code that includes function pointers and inlined assembly. This article introduces a novel technique for checking pointer safety violations, called Symbolic Object Code Analysis (SOCA), which is based on bounded symbolic execution, incorporates path-sensitive slicing, and employs the SMT solver Yices as its execution and verification engine. Extensive experimental results of a prototypic SOCA Verifier, using the Verisec suite and almost 10,000 Linux device driver functions as benchmarks, show that SOCA performs competitively to modern source-code model checkers, scales well when applied to real operating systems code and pointer safety issues, and effectively explores niches of pointer-complex software that current software verifiers do not reach.
\end{abstract}

\section{Introduction}

One challenge in verifying complex software is the proper analysis of pointer operations. A recent study shows that the majority of errors found in device drivers involve pointer safety [10]. Writing software that is free of memory safety concerns, e.g., free of errors caused by pointers to invalid memory cells, is difficult since many such issues result in program crashes at later points in execution. Hence, a statement causing a memory corruption may not be easily identifiable using conventional validation and testing tools such as Purify [45] and Valgrind [40].

Today's static verification tools, including software model checkers such as $[4,11,12,20]$, are also not of much help: they either assume that programs do "not have wild

\footnotetext{
* An extended abstract of this article appeared in the proceedings of SPIN 2010: "Model Checking Software", volume 6349 of Lecture Notes in Computer Science, pages 4-21, Springer, 2010.
}

pointers" [3], perform poorly in the presence of pointers [38], or simply cannot handle certain software. A particular challenging kind of software are operating system (OS) components such as device drivers, which are usually written in $\mathrm{C}$ code involving function pointers, pointer arithmetic, and inlined assembly. Further issues arise because of platform-specific and compiler-specific details concerning memory layout, padding, and offsets [2]. In addition, several approaches to model checking compiled programs given in assembly or bytecode $[7,27$, $35,48,52$ ] and to integrating symbolic execution [28] with model checking $[17,18,26,43,49]$ have been presented. However, these are tailored to exploit specific characteristics of certain programming paradigms such as objectoriented programming, or lack support for data structures, function pointers, and computed jumps, or require substantial manual modeling effort.

This article introduces and evaluates a novel, automated technique to identifying pointer safety violations, called Symbolic Object Code Analysis (SOCA). This technique is based on the symbolic execution [28] of compiled and linked programs. In contrast to other verification techniques, SOCA requires only a minimum of manual modeling effort, namely the abstract, symbolic specification of a program's execution context in terms of function inputs and initial heap content. Our extensive evaluation of a prototypic SOCA implementation shows that SOCA performs competitively to state-of-the-art model checkers such as SLAM/SDV [4], SatAbs [12], or BLAST [20] on programs with "well-behaved" pointers, and also that it scales well when applied to "dirty" programs such as device drivers that cannot be properly analyzed with source-code model checkers.

Technically, the SOCA technique traverses the program's object code in a systematic fashion up to a certain depth and width, and calculates at each assembly instruction a slice [53] required for checking the relevant pointer safety properties. It translates such a slice and 
properties into a bit-vector constraint problem, and executes the property checks by invoking the Yices SMT solver [15]. During program traversal, SOCA unwinds loops and follows function calls. Since address calculation and the management of stack frames is completely revealed in object code, SOCA does not require a separate reasoning to account for interprocedural effects. To the best of our knowledge, SOCA is the only program verification technique reported in the literature that features full support for pointer arithmetics, function pointers, and computed jumps, rather than the partial support offered in static analysis tools with bounded address tracking [27]. While SOCA is based on existing and wellknown techniques, combining and implementing these for object-code analysis proved challenging. Much engineering effort went into our SOCA implementation, so that it scales to complex real-world OS code such as Linux device drivers.

The particular combination of techniques in SOCA is well suited for checking pointer safety. Analyzing object code is beneficial in that it inherently considers compiler specifics such as code optimizations, makes memory layout obvious, and does away with the challenge of handling mixed input languages involving assembly code. Symbolic execution, rather than the concrete execution adopted in testing, can handle software functions with many input parameters whose values are typically not known at compile time. It is the existence of efficient SMT solvers that makes the symbolic approach feasible. Symbolic execution also implies a path-wise exploration, thus reducing the aliasing problem and allowing us to handle complex pointer operations and computed jumps. In addition, slicing is now conducted at pathlevel instead of at program-level, resulting in drastically smaller slices to the extent that abstraction is not necessary for achieving scalability. However, the price of symbolic execution is that it must be bounded and can thus only analyze code up to a finite depth and breadth.

To evaluate our technique, we have implemented a prototypic SOCA tool, the SOCA Verifier, for programs compiled for the 32-bit Intel Architecture (IA32) and performed extensive experiments. Using the Verisec [33] benchmark we show that our verifier performs on par with the model checkers LoopFrog [31] and SatAbs [12] with regards to performance, error detection, and falsepositive rates. We have also applied the SOCA Verifier to 9,296 functions taken from 250 Linux device drivers. Our tool is able to successfully analyze $95 \%$ of these functions and, despite the fact that SOCA performs a bounded analysis, $28 \%$ of the functions are analyzed exhaustively. Since heap-aware program slicing is key to SOCA's scalability, we also provide a detailed experimental evaluation of the slicing strategy implemented in our tool. Overall, SOCA proves itself to be a capable technique when being confronted with checking pointercomplex software such as OS components. It effectively explores semantic niches that neither currently available testing tools nor software model checkers reach.

The remainder of this article is organized as follows. In Sec. 2 we outline the objectives and challenges for our SOCA technique. We also provide background information on Valgrind's intermediate representation (IR), on which our tool is based. A detailed explanation of SOCA follows in Sec. 3, where we discuss the translation from our intermediate representation to bit-vector constraints for the SMT solver Yices, and explain how the constraint representation can be annotated with assertions expressing pointer safety properties. We also give a high-level explanation of our slicing algorithm and discuss the handling of register access, memory access, and computed jumps in SOCA. Sec. 4 is dedicated to a more detailed presentation of our algorithms in pseudo-code, and to an overview of the design decisions that influenced the development of our prototypical SOCA implementation, the SOCA Verifier. Our experimental results are reported in Sec. 5. We present an evaluation of the effectiveness of the SOCA Verifier for finding bugs in the Verisec benchmark suite, give details on the impact of the slicing algorithm, and analyze the SOCA Verifier's scalability based on an extensive case study of Linux device drivers. Finally, we discuss related work in Sec. 6 and our conclusions in Sec. 7.

\section{Pointer Safety, Aliasing \& IR}

The verification technique developed in this article aims at ensuring that every pointer in a given program is valid in the sense that it (i) never references a memory location outside the address space allocated by or for that program, and (ii) respects the usage rules determined by the Application Programming Interfaces (APIs) employed by the program.

\subsection{Pointer Safety}

There are six categories of pointer safety properties:

- Dereferencing invalid pointers: A pointer must not be NULL, shall be initialized, and shall not point to a memory location outside the address space allocated by or for the program;

- Uninitialized reads: Memory cells shall be initialized before they are read;

- Violation of memory permissions: Permissions to a program's segment shall be observed, which are assigned when the program is loaded into memory and determine whether a segment can be read, written, or executed;

- Buffer overflows: Out-of-bounds read and write operations to objects on the heap and stack shall not occur, as this might lead to memory corruption and give way to various security problems; 
- Memory leaks: A program shall not lose all handles to dynamically allocated memory, as this would mean that the memory cannot be deallocated anymore;

- Proper handling of allocation $\&$ deallocation: OS provided APIs for the dynamic (de)allocation of memory shall be respected, whose documentations specify precisely what pairs of functions are to be employed and how they are to be employed.

\subsection{Aliasing in Source Code $\&$ Object Code}

A major issue for analyzing pointer programs is aliasing. Aliasing means that a data location in memory may be accessed through different symbolic names. Since aliasing relations between symbolic names and data locations often arise unexpectedly during program execution, they may result in erroneous program behavior that is particularly hard to trace and debug. To illustrate this, the following $\mathrm{C}$ program shows a rather complicated way of implementing an infinite loop:

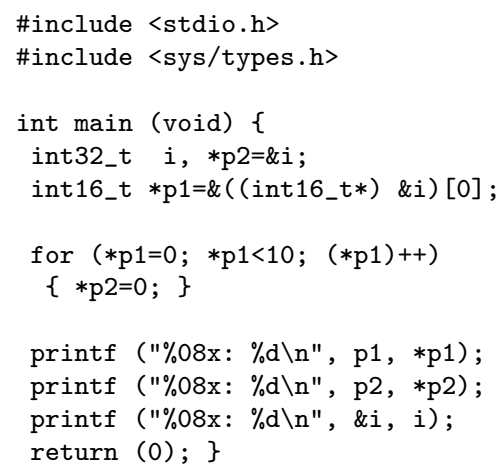

At least three different outcomes of the program's execution can occur as a result of varying assumptions made about pointer aliasing by the developer and the compiler, as well as of compiler optimizations applied to the code. In the following listing we give the output of the program when compiled with gcc version 4.1.2 (Listings (a) and (b)) and gec version 4.3.1 (Listings (c) and $(d))$ :

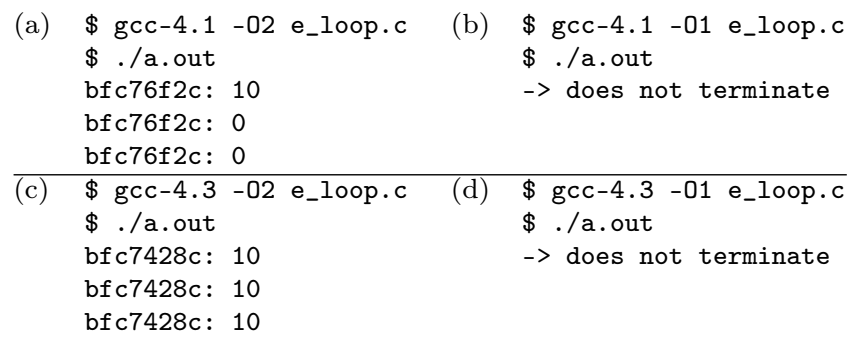

More surprises are revealed when studying the excerpt of the corresponding assembly code displayed below, which was obtained by disassembling the program that produced the output shown in Listing (c). One can see at instructions $80483 \mathrm{cf}$ and $80483 \mathrm{~d} 6$ that $p 1$ and $p 2$ are pointing to the same location and that ${ }^{*} p 2$ is actually written before ${ }^{*} p 1$. This is unexpected when looking at the program's source code but valid from the compiler's point of view, since it assumes that the two pointers are pointing to different data objects. As another consequence of this assumption, register eax is never reloaded from the memory location to which $p 1$ and $p 2$ point.

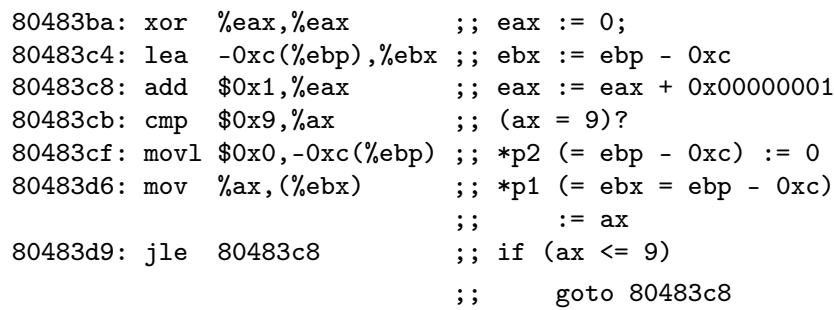

This example shows that source-code-based analysis has to decide for a particular semantics of the source language, which may not be the one that is used by a compiler. Hence, results obtained by analyzing the source code may not meet a program's runtime behavior. While this motivates the analysis of compiled programs, doing so does not provide a generic solution for dealing with pointer aliasing, as aliasing relationships may depend on runtime conditions.

Arguably, the above example program is artificial as it violates the $\mathrm{C}$ standard [8]. With respect to aliasing, the standard specifies that it is illegal for pointers of different types to reference the same memory location. In practice, however, a number of large, well-known, and extensively used software projects, including the Linux kernel, violate this part of the standard so as to facilitate the use of particular programming styles or for performance reasons. In the case of the Linux kernel, the compiler's assumptions on "strict aliasing" cause problems when optimizing inlined code.

In general, using architecture dependent and compiler dependent code is quite common in embedded and real-time systems and in low-level OS components. For example, the Linux kernel contains a substantial fraction of code that is specific to the many architectures for which the kernel may be compiled. This paradigm is even more visible in the FreeRTOS real-time OS [5], where two-thirds of the kernel comprise of architecture specific and compiler specific code, and implementations of these two-thirds are provided for 16 build environments on 31 architectures. In case of FreeRTOS, only $5 \%$ of the code base are written with no specific assumptions regarding the build environment in mind.

There is also a growing body of software whose source code is not released to the party using or re-distributing this software. To check closed-source components for conformance with, e.g., API specifications, analyzing the object code of that component is the only feasible solution.

\subsection{Intermediate Representation}

A program under analysis is stored by us in an intermediate representation (IR) borrowed from Valgrind [40], 
a framework for dynamic binary instrumentation. The IR consists of a set of basic blocks containing a group of statements such that all transfers of control to the block are to the first statement in the group. Once the block has been entered, all of its statements are executed sequentially until an exit statement is reached. An exit is always denoted as goto <target>, where <target> is a constant or temporary register that determines the next program location to be executed. Guarded jumps are written if (<condition>) goto <target>, where <condition> is a temporary register of type boolean, which has previously been assigned within the block.

The listing below depicts an example of assembly statements and their corresponding IR statements. It shows how, e.g., the xor statement is decomposed into explicitly loading (GET) the source register 0 into the temporary registers t8 and t9, performing the xor operation into the temporary register $t 7$, followed by storing (PUT) the result back. All operands used in the first block of the example are 4 bytes, or 32 bits, in size.

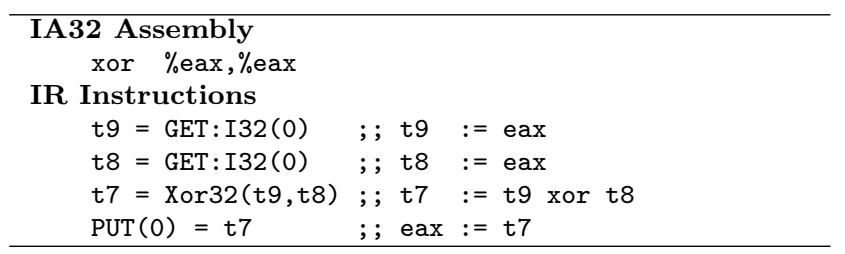

As can be seen, the IR is essentially a typed assembly language in static-single-assignment (SSA) form [34], and employs temporary registers - which are denoted as $t<n>-$ and the guest state. The guest state consists of the contents of the registers that are available in the architecture for which the program under analysis is compiled. While machine registers are always 8 bits long, temporary registers may be $1,8,16,32$, or 64 bits in length. As a result, statement t9 = GET: I32(0) means that $t 9$ is generated by concatenating machine registers 0 to 3 . Since each IR block is in static-single-assignment form with respect to the temporary registers, $t 9$ is assigned only once within a single IR block.

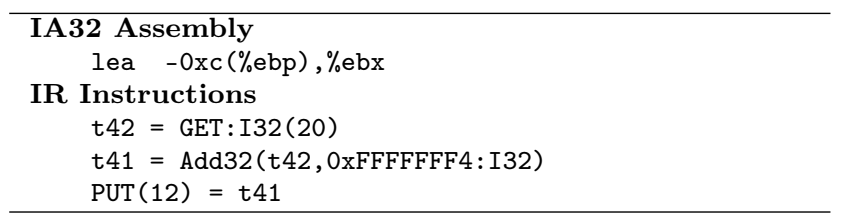

As a valuable feature for analyzing pointer safety, Valgrind's IR makes all address computations as well as load and store operations to memory cells explicit. In particular, instructions capable of performing complex address computations, such as the above lea - "load effective address", an instruction frequently used for computing offsets into arrays or structs - are decomposed into sequences of basic operations.

\section{SOCA - Symbolic Object Code Analysis}

This section introduces our novel approach to verifying pointer safety in compiled and linked programs, which we call Symbolic Object Code Analysis (SOCA). The basic idea behind our approach employs well-known techniques including symbolic execution [28], SMT solving [32], and program slicing [53]. However, combining these ideas and implementing them in a way that scales to real applications, such as Linux device drivers, is challenging and the main contribution of this article.

Starting from a program's given entry point, we automatically translate each instruction of the program's object code into Valgrind's IR language. This is done lazily, i.e., as needed, by iteratively following each program path in a depth-first fashion and resolving target addresses of computed jumps and return statements. We then generate systems of bit-vector constraints for the path under analysis, which reflect the path-relevant register content and heap content of the program. In this process we employ a form of program slicing, called pathsensitive and heap-aware program slicing (see below), which is key to SOCA's scalability and makes program abstraction unnecessary. Finally, we call the SMT solver Yices [15] to check the satisfiability of the resulting constraint systems and thus the validity of the path. This approach allows us to instrument the constraint systems on-the-fly as necessary, by adding constraints that express the desired pointer safety properties, e.g., whether a pointer points to an allocated address.

SOCA leaves most of a program's input and initial heap content unspecified in order to allow the SMT solver to search for subtle inputs that may reveal pointer errors. Obviously, our analysis by symbolic execution cannot be complete; the search space has to be bounded since the total number of execution paths and the number of instructions per path may be infinite. Our experimental results (cf. Sec. 5) show that this boundedness is not a restriction in practice; many interesting programs, such as Linux device driver functions, are relatively "shallow" and may still be analyzed either exhaustively or to an acceptable extent.

\subsection{Encoding Path Constraints in Yices}

We start off our detailed presentation of SOCA by describing the translation of IR into bit-vector constraints expressed in the input language of Yices, and the encoding of our categories of pointer safety properties as assertions in Yices.

Translating IR into Yices constraints. In order to translate IR statements into bit-vector constraint systems for Yices, we have defined a simple operational semantics for Valgrind's IR language. Doing so is rather straightforward and is detailed in the first author's PhD thesis [36]. Instead of presenting this semantics here, we 
focus directly on its application using examples that illustrate our translation.

As a first example we consider statement PUT (0) = t7 of Sec. 2.3. Intuitively, the semantics of PUT is to store the value held by $t^{r} 7$ to the guest state, in registers 0 to 3 (i.e., r0 to $r 3$ below):

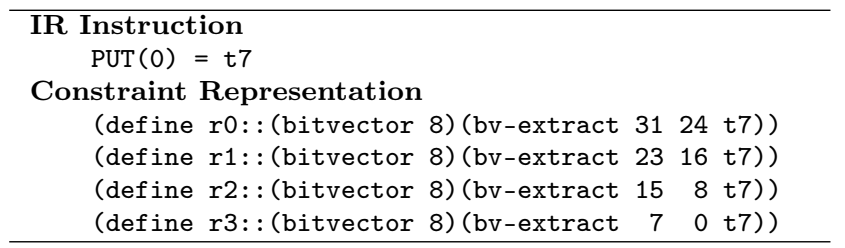

Here, the bv-extract operation denotes bit-vector extraction in Yices. Note that the IA32 CPU registers are assigned in reverse byte order, while arithmetic expressions in Yices are implemented for bit-vectors that have their most significant bit at position 0 . Since access operations to the guest state may be $8,16,32$, or 64 bit aligned, we have to translate the contents of temporary registers when accessing the guest state.

Similar to the PUT instruction, we can express GET, i.e., the loading of a value from the guest state, as the concatenation of bit-vectors, and the Xor and Add instructions in terms of bit-vector arithmetic. Note that hexadecimal literals in the IR have been converted to decimal numbers in the constraint representation, i.e. $\mathrm{OxFFFFFFF}_{16}=4294967284_{10}$ :

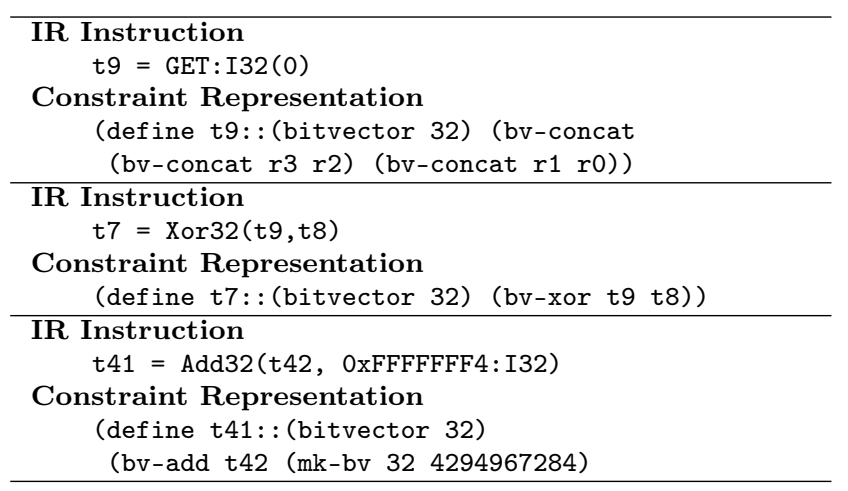

More challenging to implement are the IR instructions ST (store) and LD (load), which facilitate memory access. The main difference of these instructions to PUT and GET is that the target of ST and the source of LD are variable and may only be computed at runtime. To include these statements in our framework we have to express them in a flexible way, so that the SMT solver can identify cases in which safety properties are violated. In Yices we declare a function heap as our representation of the program's memory:

(define heap: :(-> (bitvector 32) (bitvector 8)))

To stay as close as possible to the operational semantics of IA32 CPU instructions, our heap function provides a mapping from bit-vectors of length 32, i.e., 4byte wide addresses, to bit-vectors of length 8 , i.e., 1 byte aligned memory cells. An exemplary ST statement
$\mathrm{ST}(\mathrm{t} 5)=\mathrm{t} 32$ can be expressed in terms of updates of that function:

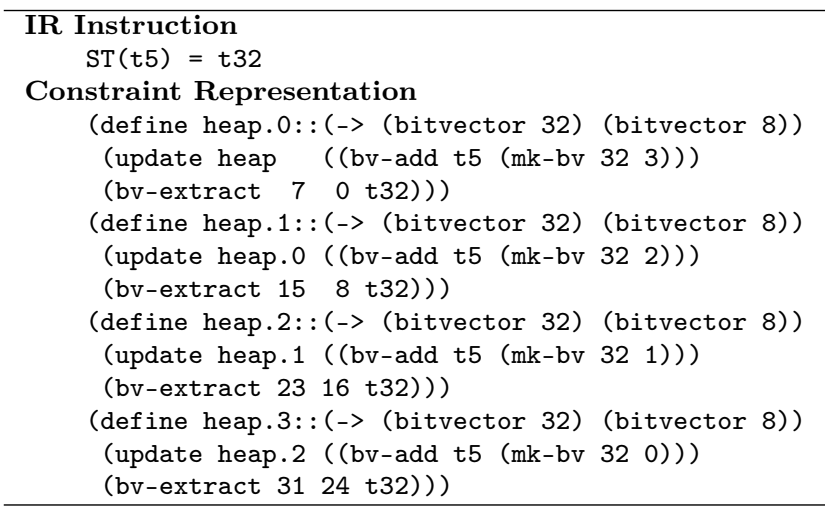

Since the above ST instruction stores the content of a $32-$ bit variable in four separate 8-bit memory cells, we have to perform four updates of heap. Byte-ordering conventions apply in the same way as explained for PUT. Constraints for the LD instruction are generated analogously:

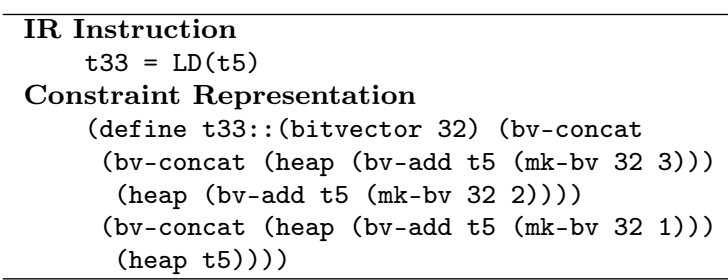

Here, 133 is assigned the concatenation of the bit-vectors obtained by applying heap to the addresses $t 5+3, t 5+2$, $t 5+1$ and $t 5$.

Encoding pointer safety properties. Being able to translate each object-code instruction into constraints allows us to express the pointer safety properties of Sec. 2.1 in terms of assertions within the constraint systems.

The simplest case of such an assertion is a null-pointer check. For the ST instruction in the above example, we state this assertion as (assert $(=t 5(m k-b v 320)))$. If the resulting constraint system is satisfiable, Yices will return a possible assignment to the constraint system variables representing the program's input. This input is constructed such that it will drive the program into a state in which $t 5$ holds the value null at the above program point.

However, many pointer safety properties demand additional information to be collected about the program's current execution context. In particular, answering the question whether a pointer may point to an "invalid" memory area requires knowledge of which cells are currently allocated. We retain this information by adding a function named heaploc to our memory representation:

(define heaploc::(-> (bitvector 32) (record alloc::bool init: :bool start: (bitvector 32) size: (bitvector 32) api::int perms::int)))

As can be seen, our heaploc function implements a mapping from bit-vectors of size 32 , i.e., addresses, to records 
that contain the fields alloc, init, start, size, api, and perms. These fields denote whether a heap cell is allocated, whether it is initialized, the start address and size of the heap chunk the cell belongs to, the OS API that was used to allocate the memory, and the memory permissions currently assigned to the cell, respectively. To reduce the size and search space of the resulting constraint systems we check assertions one-by-one with a reduced heaploc function for each property. For example, the heaploc function allows us to express assertions stating that, e.g., pointer $t 5$ has to point to an allocated address at the program location where it is dereferenced, as:

(assert (= (select (heaploc t5) alloc) true))

When checking a series of assertions such as the above, e.g., for a number of consecutive store operations, we employ a reduced heaploc function that is a mapping from addresses to a record containing the alloc field only.

The other pointer safety properties, as mentioned in Sec. 2.1, may be encoded along the lines of the above. In particular, we can check whether all heap cells read or written by a single LD or ST instruction, respectively, belong to the same chunk in memory:

(assert ( $=$ (select (heaploc t5) start)

(select (heaploc (bv-add t5 (mk-bv 32 1))) start)))

Here, we assume that the address stored in $t 5$ will be dereferenced to load a 16 -bit value from the 8-bit aligned memory. Hence, we should check whether the memory cells addressed by $t 5$ and $t 5+1$ belong to the same object on the heap. In a similar manner, we can verify that heap cells dereferenced in different iterations of the same loop are pointing to the same memory chunk. Violations of these properties indicate potential buffer overflow bugs for which we issue, however, warning messages only. This is because read and write operations spanning multiple objects in memory are sometimes intentional and do not necessarily imply an immediate failure of the program under analysis.

Expressing other pointer safety properties often requires a more complete heaploc function. This may involve information about whether a particular memory chunk is statically allocated, or whether an OS API function such as malloc() has been used to allocate heap space at run-time. The enlarged heaploc function may then be employed to check API usage rules, i.e., for verifying that a program only attempts to free() dynamically allocated heap locations, and that the correct API functions are called for each chunk. The full details of our generation of constraint systems can be found in [36].

\subsection{Path-Sensitive Slicing}

To ensure scalability of our SOCA technique we do not run Yices on an entire path's constraint system. Instead we compute a slice [53] of the system containing only those constraints that are relevant to the pointer safety property to be checked at a particular program location.

The approach to path-sensitive program slicing in SOCA employs an algorithm based on system dependence graphs as introduced in [22]. Our slices are extracted using conventional slicing criteria (L, var), denoting a variable var that is used at program location $L$. However, in contrast to [22], we extract the slice over the single path currently being analyzed instead of the program's entire control flow. The slice is then computed by collecting all statements on which var is data dependent by tracing the path backwards, starting from $L$ up to the program's entry point. While collecting flow dependencies is relatively easy for programs that do only use CPU registers and temporary registers, it becomes difficult when dependencies to the heap and stack are involved, as is discussed in the following.

\section{Handling memory access in slicing. Consider the two} IR statements:

$01 \quad \mathrm{ST}(\mathrm{t} 5)=\mathrm{t} 32$;

$02 \mathrm{t} 31=\mathrm{LD}: \mathrm{I} 32(\mathrm{t} 7)$

To compute a slice for the slicing criterion $(02, t 31)$ we must know whether the store statement ST may affect the value of $t 31$, i.e., whether $t 5$ and $t 7$ may alias. We obtain this information by using Yices to iteratively explore the potential address ranges that can be accessed via $t 5$ and $t 7$, respectively. Aliasing might then occur if the address ranges of $t 5$ and $t 7$ overlap.

We explore the address range of, e.g., $t 5$ by making Yices find an assignment $e$ for $t 5$ such that the constraint system generated from slice $(01, t 5)$ is satisfied. When reading $e$, which is represented by Yices as a bitvector, we compute its integer representation and further satisfying assignments $e^{\prime}$, i.e., models of the constraint system, such that $e>e^{\prime}$ or $e<e^{\prime}$ holds, until the range is explored. To use Yices as efficiently as possible when searching for satisfying models, we employ stepwise adding or retracting of constraints. The pseudo-code for this algorithm is given as function compute target addresses in Table 2. By computing the potential address range accessed by a pointer used in a load statement, $t 7$ in our example, and looking for memory intervals overlapping with the range of $t 7$, we can now determine which store operations may affect the result of the load operation.

Observe that, since we remember only the "maximal" and "minimal" satisfying models for a given pointer, the above calculation results in an over-approximation because not the entire address range may be addressable by that pointer. However, using this abstraction presents a trade-off concerning only the size of the computed slices and not their correctness, and helps us to keep the number of calls to Yices and the amount of data to be stored small. Despite being conservative when computing address ranges, our experience shows that most memory 


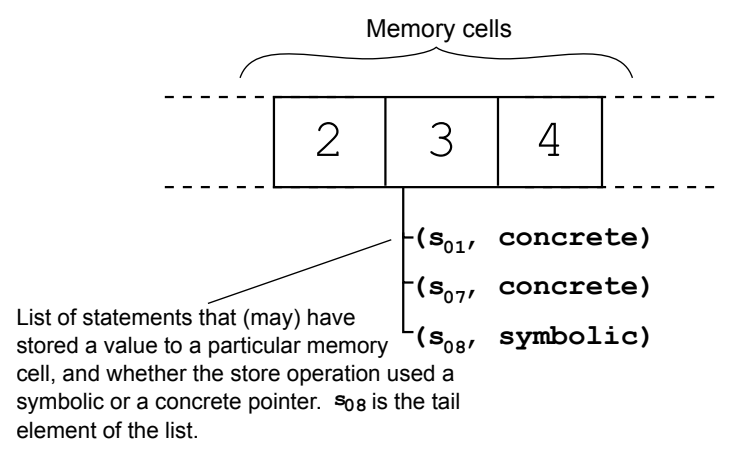

Figure 1. Illustration of SOCA's memory model.

access operations end up having few dependencies; this is because most pointers evaluate to a concrete value, i.e., the constraint system has exactly one satisfying model, rather than a symbolic value that represents potentially many concrete values.

Representing a path's memory state. As illustrated in Fig. 1, SOCA's internal representation of a path's memory state is that of an array of memory cells. For each memory cell we remember a list of those statements on the path that may have stored a value to the memory cell. The tail element of this list denotes the most recent store operation to the cell. We also keep track of whether a cell was accessed through a symbolic or concrete pointer. A symbolic pointer is a pointer whose exact value cannot be determined at the point at which it is dereferenced during symbolic execution. This usually happens when the entire pointer or some component of it (e.g., its base or offset) is retrieved from an incompletely specified component of the execution environment, or directly from the input to the analyzed program.

The information of whether a store operation was performed via a symbolic pointer or a concrete pointer is used by our slicing algorithm to reduce the sizes of slices as follows. When tracing the data dependencies of a load statement that accesses a particular memory cell, we first obtain the list of statements that may have stored data to that cell. The list is traversed from tail to head. All store statements from the list are added to the slice, until a store statement that used a concrete pointer is found. This is sound since we always add all symbolic stores that may have modified the content of the memory cell, together with the most recent concrete store that has definitely stored content to that cell. As we show in the experimental evaluation of our slicing algorithm in Sec. 5.2, our method reduces the sizes of path-sensitive slices by one order of magnitude on average.

Handling computed jumps. One challenge when analyzing compiled programs arises from the extensive use of function pointers and jump target computations. While most source-code based approaches simply ignore function pointers $[4,12,20]$, this cannot be done when analyz- ing object code since jump computations are too widely deployed here. The most common example for a computed jump is the return statement in a subroutine. To perform a return, the bottom element of the stack is loaded into a temporary register, e.g., $t 1$, followed by a goto $t 1$ statement that effectively sets the value of the program counter to $t 1$. Further examples for computed jumps are jump tables and function pointers. In our approach, jump target addresses are determined in the same way as addresses for load and store instructions, i.e., by computing a slice for each jump target and then using Yices to determine satisfying models for the target register.

Optimizing GET \& PUT statements. A potential problem with respect to the scalability of our approach arises from the vast number of GET and PUT statements in IR code. In particular, the frequent de-/re-composing of word-aligned temporary registers into guest registers and back into temporary registers raises the number of I/O operations performed by our prototypic implementation of SOCA. That is, we have to generate constraints for these operations and pass these constraints to Yices. This, in turn, increases the time required by Yices to parse the constraint systems and might also result in additional variables in the decision procedure. By employing single-instruction basic blocks that can be used as a jump target from multiple different origins, e.g. in a different iteration of a loop, we avoid the need to repeat the expensive translation from object code to IR and further on to constraints whenever an instruction is used in a different execution context. However, this is at the expense of having to deal with a larger number of IR instructions due to loading and storing the guest state at the beginning and end of each IR block, which in turn results in larger constraint systems.

An efficient way around this issue is to eliminate unnecessary GET and PUT operations, based on a reaching definition analysis for a given register and path. We can apply the same optimizations to memory accesses in cases where the address arguments to LD and ST evaluate to constant values. Dealing with unnecessary GET, PUT, LD, and ST statements - by performing the above optimizations on IR level for an entire execution path - results in smaller constraint systems and shorter runtimes of SOCA and Yices, as reported in Sec. 5.2. We refer to the above two optimizations as the GETESPUT strategy and the Memory strategy, respectively.

Determining a valid initial memory state. Another challenge when implementing symbolic execution as an SMT problem is given by the enormous search space that may result from leaving the program's initial memory state and heap state undefined. OS components, including functions taken from device drivers, make regular use of an external data environment consisting of heap objects allocated and initialized by other OS modules. 
Table 1. An example: C code fragment and IR

\begin{tabular}{|c|c|c|}
\hline C Code & \multicolumn{2}{|c|}{ IR Instructions } \\
\hline $\mathrm{x}=2 ;$ & $0 \times 01$ & store $(3)=2$ \\
\hline while $(\mathrm{x} \quad !=0)$ & $0 \times 02$ & to $=\operatorname{load}(3)$ \\
\hline\{ & $0 \mathrm{x} 03$ & $t 1=\operatorname{sub}(t 0,0)$ \\
\hline & $0 \times 04$ & t2 $=$ calculate_condition $(\mathrm{Z})$ \\
\hline & $0 \times 05$ & if t2 goto $0 \mathrm{xOA}$ \\
\hline $\mathrm{x}--$; & $0 \mathrm{x} 06$ & $t 3=\operatorname{sub}(t 0,1)$ \\
\hline & $0 \times 07$ & store $(3)=t 3$ \\
\hline$* \mathrm{y}=\mathrm{z}$; & $0 \mathrm{x} 08$ & store $(t 5)=t 6$ \\
\hline$\}$ & $0 \times 09$ & goto $0 \times 02$ \\
\hline return; & $0 \mathrm{xOA}$ & return \\
\hline
\end{tabular}

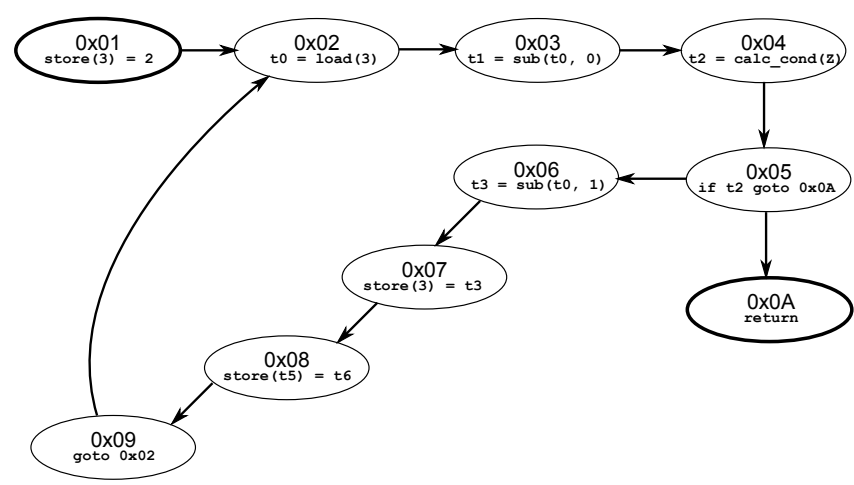

Figure 2. An example: Control flow graph obtained from the IR code of Table 1.

Hence, this data environment cannot be inferred from the information available in the program binary. In practice, data environments can often be embedded into our analysis without much effort, by adding a few lines of $\mathrm{C}$ code as a preamble to the source code to be analyzed, as is shown by us in [39].

\section{The SOCA Algorithms in Detail}

In this section we present all details of our SOCA program analysis by showing it in action on a small example, and by providing commented pseudo code. We also describe the architecture and major design and implementation decisions behind our prototypic implementation of the SOCA technique, the SOCA Verifier.

\subsection{Example Program \& Its Internal Representation}

The running example for this section is the program fragment given in $\mathrm{C}$ and IR code in Table 1, which contains a while-loop counting from 2 down to 0 . In each iteration, after decrementing counter $\mathrm{x}$, the memory cell pointed to by $\mathrm{y}$ is assigned $\mathrm{z}$. Both $\mathrm{y}$ and $\mathrm{z}$ are expected to be assigned before execution. To show how our analysis deals with symbolic pointer values, we assume that $\mathrm{y}$ and $\mathbf{z}$ are partially dependent on the program's input such that the address range accessible via y symbolically overlaps with the location at which the value of $\mathrm{x}$ is stored.

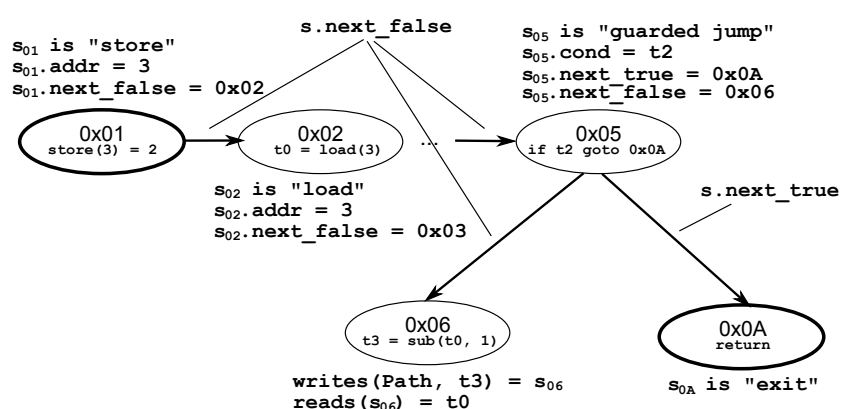

Figure 3. An example: SOCA's internal representation of the program of Table 1

The IR code given in Table 1 has been simplified in the following ways to improve readability over Valgrind's IR: ( $i$ ) we do not show any types but assume that all temporary registers are one byte long; (ii) we simply write load and store to denote load and store instructions that operate on memory cells that are also one byte long; (iii) we obtain the content of the CPU's Z flag-register with calculate_condition(Z). The Z flag specifies whether the result of the last arithmetical operation was zero. Our example program uses this in combination with the sub instruction at address $0 \times 03$ to evaluate the condition of the while loop.

Fig. 2 shows the control flow graph of the example program, which is the input to our program analysis. As can be seen, each IR statement of Table 1 is represented by a node in the graph. Of course, due to computed jumps, it is usually not possible to statically compute the control flow of an entire program in practice. Hence, the SOCA Verifier computes the control flow iteratively, decoding and analyzing one CPU instruction at a time.

Our data structure encoding a particular statement, e.g., a node in the control flow graph, is illustrated in Fig. 3 on our small example of Table 1 . If statement $s$ is a guarded jump, i.e., an if <condition> goto <target> construct of Valgrind's IR, we write s.cond to access the condition-variable and s.next true to access the target. Each statement contains a field s.next_false, which points to the statement's default successor. If a statement is a load or store operation, we write s.addr to denote the address variable holding the memory address used by the statement. In addition, $s$ also has the members s.true_visited and s.false_visited, which are used as markers for backtracking on a path. Since these components hold dynamic data indicating the progress of our analysis, they are not presented in the static control flow in Fig. 3. However, they are employed by function traverse in Table 3. 


\subsection{Pseudo Code}

In the pseudo code presented in Tables 2 and 3 we focus on illustrating SOCA's iterative program exploration and our slicing technique. Some details, such as the actual constraint generation and optimization, caching strategies, handling timeouts and the exhaustion of analysis bounds, and how constraints are iteratively added and retracted in Yices, have been left out. However, for readability we provide interfaces for the functions implementing these features.

The core of our SOCA technique is the systematic traversal of a program as implemented by functions traverse and traverseCore in Table 3, where traverse(Prog, s) recursively generates all paths of program Prog starting from statement $s$. Here, Prog is a directed graph of statements, which represents the program's control flow and in which each statement has at most two successors (we do not consider computed jumps in the pseudo code).

Since the traversal of a program has to be bounded, our implementation of SOCA is highly configurable with respect to the bounds supported. Initially, we implemented relatively naive bounds such as defining a maximum path length, a maximum number of paths to be explored, and a timeout for the SMT solver. While experimenting with the SOCA Verifier we found alternative bounds extremely useful. In particular, we use the number of times a particular program statement may be unrolled per path and over all paths, to effectively guide our analysis. Also coverage criteria such as branch coverage have been implemented to limit the number of times guarded jumps may be exercised. Since the implementation of bounds is straightforward, we do not present it in the pseudo code.

More interesting is the pseudo code for SOCA's slicing algorithm in function slice(Path, var), also in Table 3. The input to slice is a program path Path and a slicing criterion in terms of a variable var. Since all variables in the program path, i.e., register names and temporary register names, have been renamed to be in static-single-assignment (SSA) form, all variable names are unique. Hence, we can consider Path to be a set of statements. Each statement $s$ may read a number of variables that are assigned by other statements executed before $s$, and may assign new variables. The slicing algorithm employs the function reads $(s)$ to obtain the set of variables that are read by $s$. We further write $s:=$ writes (Path, var) to yield the uniquely defined statement $s \in$ Path that assigns variable var.

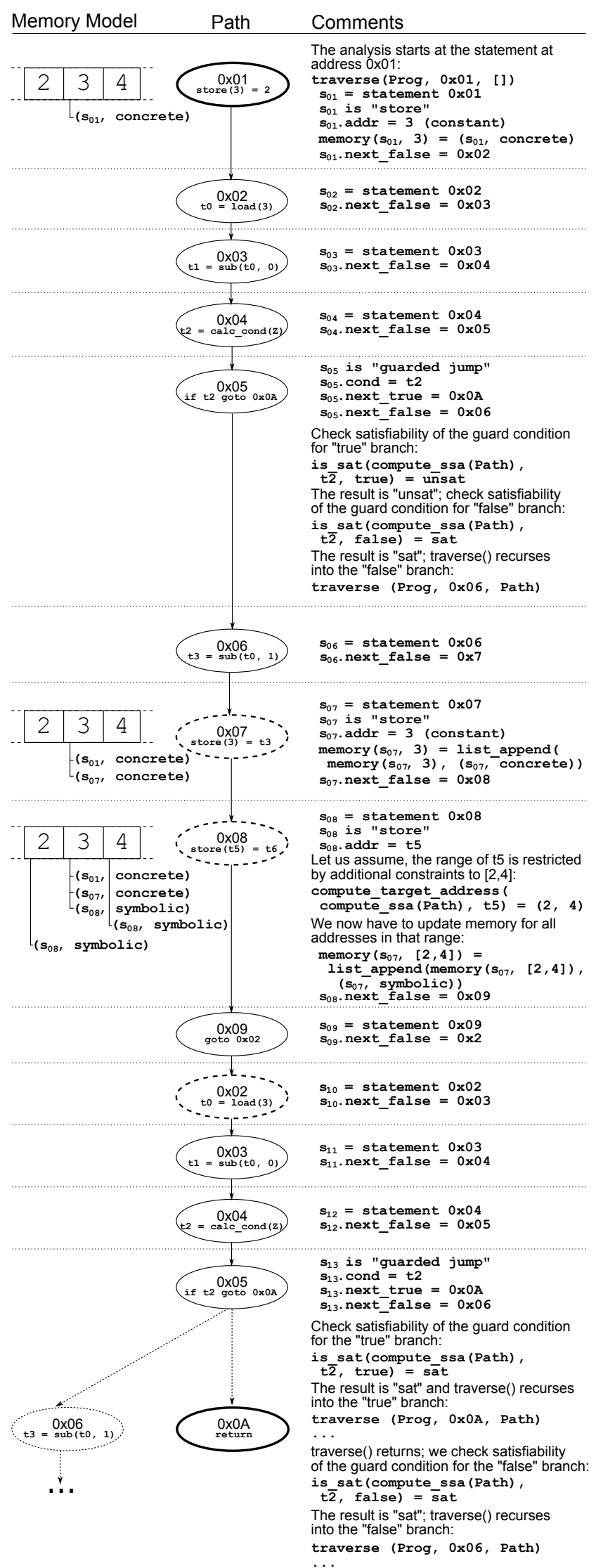

Figure 4. An example: Application of function traverse. Checks of pointer safety properties are to be performed in the dashed nodes. 
Table 2. Pseudo code of the SOCA algorithm (Part I)

\begin{tabular}{|c|c|}
\hline \multirow{3}{*}{$\begin{array}{l}\text { Helper Functions. } \\
\text { list_append (e, L) appends e as the new tail element of } \mathrm{L} \text { and } \\
\text { returns the resulting list. }\end{array}$} & \\
\hline & \multirow{2}{*}{$\begin{array}{l}\text { reads }(\mathrm{s}) \text { returns the set of variables that are read by statement } \\
\mathrm{s} \text {. } \\
\text { Function reads }\end{array}$} \\
\hline & \\
\hline \multirow{3}{*}{$\begin{array}{l}\text { Function list_append } \\
\text { Input e: Element, } \\
\text { L: List; } \\
\text { Output List; }\end{array}$} & Input s: Statement; \\
\hline & Output Set of Variable; \\
\hline & $\overline{\text { is_sat }(\mathrm{P}, \mathrm{g}, \mathrm{b}) \text { invokes Yices to check whether assertion }(\mathrm{g}}$ \\
\hline \multirow{2}{*}{$\begin{array}{l}\text { list_remove_tail(L) removes the last element from list } \mathrm{L} \text { and } \\
\text { returns the resulting list. }\end{array}$} & of $(P, g)$ \\
\hline & Function is_sat \\
\hline \multirow{4}{*}{$\begin{array}{l}\text { Function list_remove_tail } \\
\text { Input L: List; } \\
\text { Output List; }\end{array}$} & Input P: Set of Statement, \\
\hline & g: Guard Variable, \\
\hline & b: Bool; \\
\hline & Output Bool; \\
\hline \multirow{4}{*}{$\begin{array}{l}\text { list_tail(L) returns the last element of list L. } \\
\text { Function list_tail } \\
\text { Input L: List; } \\
\text { Output Element of L; }\end{array}$} & $\begin{array}{l}\text { declare C: Constraint System; } \\
\mathrm{C}:=\text { compute constraint sustem }(\text { slice }(\mathrm{P}, \mathrm{g})) \text {; }\end{array}$ \\
\hline & return yices_sat $(\mathrm{C} \cup \mathrm{n}(\mathrm{g}=\mathrm{b}) ")$; \\
\hline & \\
\hline & verify_properties (s, Path) employs our memory model and \\
\hline \multirow{2}{*}{$\begin{array}{l}\text { yices_sat(C) invokes Yices on the constraint system } \mathrm{C} \text { and re- } \\
\text { turns the satisfiability result. }\end{array}$} & statement $\mathbf{s} \in$ Path as discussed in Sec. 3.2 . \\
\hline & Function verify_properties \\
\hline \multirow{3}{*}{$\begin{array}{l}\text { Function yices_sat } \\
\text { Input C: Constraint System; } \\
\text { Output sat/unsat; }\end{array}$} & Input $\mathrm{s}$ : Statement, \\
\hline & Path: Set of Statement; \\
\hline & Output ErrorMessage; \\
\hline \multirow{6}{*}{$\begin{array}{l}\text { yices_model(C) invokes Yices on the constraint system } \mathrm{C} \text { and } \\
\text { returns the satisfiability result. If available, yices_model also } \\
\text { returns an assignment for the variables of } \mathrm{C} \text { such that } \mathrm{C} \text { is } \\
\text { satisfied. } \\
\text { Function yices_model } \\
\text { Input C: Constraint System; } \\
\text { Output Tuple of (sat/unsat, model); }\end{array}$} & Memory Representation. \\
\hline & memory $(\mathrm{s}, \mathrm{a})$ represents the memory model of the SOCA \\
\hline & \\
\hline & the list denote the statements that have possibly stored con- \\
\hline & tent to memory location a at statement $\mathbf{s}$, and whether these \\
\hline & store operations have used a symbolic pointer. The tail of the \\
\hline \multirow{2}{*}{$\begin{array}{l}\text { compute_constraint_system }(\mathrm{P}) \text { generates a constraint system } \\
\text { for Yices given a program path P in SSA form. }\end{array}$} & \\
\hline & Function memory \\
\hline \multirow{2}{*}{$\begin{array}{l}\text { Function } \text { compute_constraint_system } \\
\text { Input P: Set of Statement; } \\
\text { Output Constraint System; }\end{array}$} & Input $\mathrm{s}$ : Statement, \\
\hline & $\begin{array}{l}\text { a: Address; } \\
\text { Output List of Tuples of (stat: Statement, } \\
\text { symb: symbolic|concrete); }\end{array}$ \\
\hline \multirow{2}{*}{$\begin{array}{l}\text { compute_ssa }(\mathrm{P}) \text { renames all variables in program path } \mathrm{P} \text {, which } \\
\text { is given as a list of program statements so that the variables } \\
\text { are in SSA form. }\end{array}$} & Address Computation. \\
\hline & compute_target_addresses $(\mathrm{P}, \mathrm{v})$ iteratively invokes Yices to \\
\hline \multirow{3}{*}{$\begin{array}{l}\text { Function compute_ssa } \\
\text { Input P: List of Statement; } \\
\text { Output Set of Statement; }\end{array}$} & $\begin{array}{l}\text { compute the minimal and maximal satisfying models of a con- } \\
\text { straint system generated from a slice of }(P, v) \text {. }\end{array}$ \\
\hline & Function compute_target_addresses \\
\hline & Input P: Set of Statement, \\
\hline$\overline{\text { writes }(\mathrm{P}, \mathrm{v}) \text { returns the statement in } \mathrm{P} \text { that assigns variable }}$ & v: Address Variable; \\
\hline v. Program path P is in SSA form, whence there can only be & $\begin{array}{l}\text { Output Tuple of (Address, Address); } \\
\text { declare S: Set of Statement; }\end{array}$ \\
\hline & declare C: Constraint System; \\
\hline Function writes & declare a, t: Tuple of (sat/unsat, model); \\
\hline $\begin{array}{l}\text { Input } \mathrm{P} \text { : Set of Statement, } \\
\text { v: Variable; }\end{array}$ & $\begin{array}{l}\text { declare } \min , \max : \text { Address; } \\
\mathrm{S}:=\operatorname{slice}(\mathrm{P}, \mathrm{v})\end{array}$ \\
\hline Output Statement; & $\mathrm{C}:=$ compute_constraint_system $(\mathrm{S})$; \\
\hline
\end{tabular}


Table 3. Pseudo code of the SOCA algorithm (Part II)

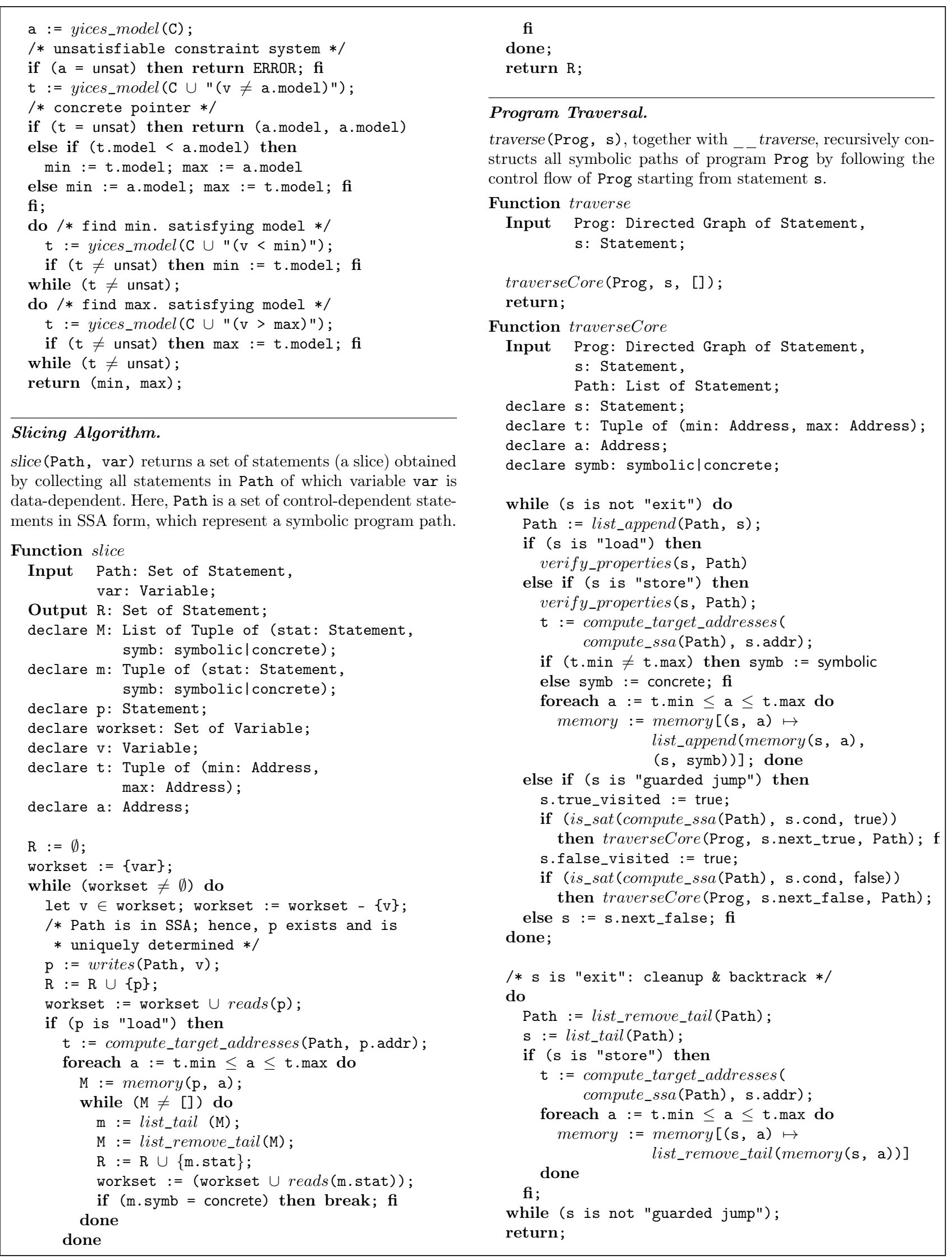


As discussed in Sec. 3.2, the SOCA technique relies on a sophisticated memory model to keep track of all store operations executed on a particular program path. In the pseudo code, this memory model is provided by function memory(statement, address). This function is updated by traverse whenever a store statement is unrolled in a path, and is used by slice to trace the data dependencies of load statements in a path.

\subsection{Illustration of a SOCA Analysis Run}

In Fig. 4 we show how traverse processes the example program of Table 1 up to the decision point at instruction $0 \times 05$ in the second iteration of the loop. At this point, depending on the value of $t 6$, guard condition $t 2$ may be satisfiable for both branches of the if statement. This results in traverse recursively evaluating the jump to the return instruction at $0 \mathrm{x} 0 \mathrm{~A}$, then recursing into the false branch at $0 \times 06$, and repeating this behavior until the analysis bounds are exhausted.

\subsection{Tool Engineering}

To evaluate our SOCA technique regarding its ability to identify pointer safety issues and to judge its performance when analyzing OS components, we have implemented SOCA in a prototypic tool, the SOCA Verifier. In this section we present details of the SOCA Verifier's architecture and discuss implementation and design decisions, including optimizations.

Architecture. The current implementation of the SOCA Verifier runs on Linux on the IA32 architecture. It is primarily designed for analyzing compiled and linked binary programs given in the Executable and Linking Format (ELF) [50] for the IA32 architecture. ELF is the standard binary format used in Linux and most other Unix derivatives. The SOCA Verifier is written in C, mainly for facilitating integration with Valgrind's VEX library [40,51]. The tool comprises about 20,000 lines of code (LOC) and took more than one person-year to build. An overview of our tool's architecture is given in Fig. 5. The figure shows the SOCA Verifier's core components, i.e., the Program Flow Analyzer, the PathSensitive Slicer, the Constraint Generator, and the Constraint Optimizer. We interface with three external components that are used for parsing binary program files in the ELF format (libELF [30]), for translating CPU instructions into IR (Valgrind's VEX Library [51]), and for solving bit-vector constraint problems (Yices [15]).

The Program Flow Analyzer is the central component of our tool. It consists of approx. 5,000 LOC, implementing the systematic traversal of the object code in a depth-first manner and passing every instruction reachable from a given program entry point to the VEX library in order to obtain its IR. The Flow Analyzer

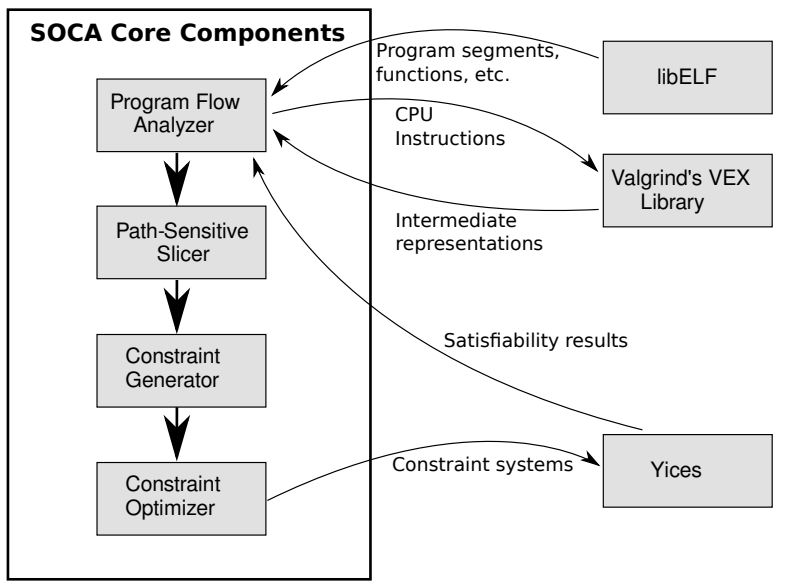

Figure 5. Architecture of the SOCA Verifier, showing the interaction between the tool's core components and its external interfaces.

then identifies control dependencies and data dependencies for each IR statement based on traditional data flow analysis [41], and generates assertions for branching conditions and pointer dereferences. The variable names of the IR and the assertions are then used as slicing criteria by the Path-Sensitive Slicer.

The Path-Sensitive Slicer consists of approx. 1,500 LOC and computes a path-slice for a slicing criterion with respect to the path currently being analyzed. Slices are passed to the Constraint Generator and further to the Constraint Optimizer, which transform the IR statements of a slice into bit-vector constraints for Yices as explained in Sec. 3.1. These two components are the biggest part of the SOCA Verifier, consisting of approx. $6,000 \mathrm{LOC}$, which is due to the multitude of different IR instructions that have to be translated into constraints.

For the purpose of analyzing operating system components, our implementation of the Constraint Generator is fairly complete with respect to the supported IR statements. We currently deal with 90 out of about 110 instructions commonly used in optimized device driver binaries. Floating point arithmetic - which is not used within the Linux kernel -, operations working on 64-bit registers, and CPU extensions recently integrated into IA32 processors for multimedia acceleration, are largely unsupported at the moment. However, with the existing SOCA tool framework, implementing a new CPU instruction usually requires less than $30 \mathrm{LOC}$ and can be done within hours. Hence, the SOCA Verifier can easily be completed and even extended to cope with new application domains such as analyzing application level programs rather than OS components.

Portability. All external components of the SOCA Verifier - libELF, Valgrind, and Yices - are available for a number of computer architectures, supporting our objective to design a verifier that can be easily adapted to check programs for platforms other than IA32. Indeed, 
we have recently started a case study on employing the SOCA Verifier to ARMv7 binaries [37]. Since Valgrind's VEX library already provides support for the ARM instruction set, implementing the port required us to facilitate parsing of a new binary format and to provide semantics for a small number of additional IR instructions only. This was achieved in less than two person-weeks of programming effort.

In a similar manner, the SOCA Verifier can be modified and extended to analyzing programs or drivers for other OSs, e.g., Microsoft Windows, or further CPU architectures, e.g., 64-bit Intel or AMD processors. However, with respect to performance and scalability, our experimental results of Sec. 5.3 indicate that further research may be required to handle a 64-bit address space efficiently. Thus, the domain in which the SOCA technique is directly and effectively applicable is in embedded systems rather than in commodity OSs for the latest off-the-shelf hardware.

Further design $\&$ implementation decisions. The different core components of the SOCA Verifier are connected by a few commonly used data structures. Most importantly, these are the control flow graph of the program under verification, the list of statements representing the program path that is currently explored, and the memory representation. Instead of generating a separate program dependency graph, we "annotate" the control flow graph and the path representation with data dependency information. In the same way, the memory representation holds direct references to nodes of the control flow graph and the path representation. This design has been chosen to reduce the memory consumption of our Verifier and to speed up searches when computing slices, at the expense of not having loosely coupled components.

Another important design decision we have made is that of caching as many intermediate results as possible. This includes the ranges of memory cells addressable by the pointers explored along a path, assignments of guard variables, and concrete (i.e., non-symbolic) data stored in particular memory cells. We also avoid the expensive re-computation of constraints. Instead, those are stored within the path representation. Of course, memory consumption is an important factor for the performance of a verification tool, and excessive caching might lead to memory exhaustion before a program is explored to a desired depth. From our experience with the SOCA Verifier, however, we have learned that the memory requirements of Yices are substantially higher than that of the other tool components. In fact, the overall size of our caches always stays below $100 \mathrm{MB}$, while the memory consumption of Yices typically remains below $3 \mathrm{~GB}$, the memory available in a modern PC. The complexity of our decision procedure is exponential in time and memory in the number of boolean variables in a constraint system. Hence, having 100 MB more memory available
Table 4. Comparison of SatAbs, LoopFrog, and SOCA

\begin{tabular}{l|c|c|c}
\hline & $R(d)$ & $R(f)$ & $R(\neg f \mid d)$ \\
\hline SatAbs (from [33]) & 0.36 & 0.08 & $\mathrm{n} / \mathrm{a}$ \\
LoopFrog (from [31]) & 1.0 & 0.26 & 0.74 \\
\hline SOCA & 0.66 & 0.23 & 0.81 \\
\hline
\end{tabular}

for Yices would not have a big impact with respect to enabling a larger depth when exploring programs.

\section{Experimental Results}

This section reports on the extensive experiments we conducted in applying the SOCA Verifier to a benchmark suite for software model checkers and to a large set of Linux device driver functions. We also present experimental results on evaluating the impact of different slicing strategies on the performance of the Verifier. Our experiments were carried out on a modern AMD Opteron 16-core PC with $2.3 \mathrm{GHz}$ clock speed and 256 GB of RAM, running 16 instances of the SOCA Verifier in parallel. However, an off-the-shelf PC with 4 GB of RAM is sufficient for the Verifier's everyday use.

\subsection{Experiments I: The Verisec Benchmark}

To enable a qualitative and quantitative comparison of the SOCA Verifier at object-code level to common tools at source-code level, we applied it to the Verisec benchmark [33]. Verisec consists of 298 test programs for buffer overflow vulnerabilities, taken from various open source programs. 149 of these programs are faulty, i.e., positive test programs. The remaining 149 are the corresponding fixed programs, i.e., negative test programs. These test cases are given in terms of $\mathrm{C}$ source code, which we compiled into object code using $g c c$ (version 4.3.1 with "O1" optimizations), and are provided with a configurable buffer size that we assigned to 4 . The bounds for the SOCA Verifier were set to a maximum of 100 paths to be analyzed, where a single instruction may appear at most 500 times per path. Yices was configured to a timeout of 300 seconds per invocation. Of these bounds, only the timeout for Yices was occasionally reached.

In previous work $[31,33]$, Verisec was used to evaluate the C-code model checkers SatAbs [12] and LoopFrog [31]. To enable a transparent comparison, we adopt the metrics proposed in [58]; in Table 4 we show the detection rate $R(d)$, the false-positive rate $R(f)$, and the discrimination rate $R(\neg f \mid d)$. The latter is defined as the ratio of positive test cases for which an error is correctly reported, plus the negative test case for which the error is correctly not reported, to all test cases. Hence, tools are penalized for not finding bugs and for not reporting a sound program as safe. 
Table 5. Performance statistics for the Verisec suite

\begin{tabular}{|c|c|c|c|c|c|c|}
\hline & average & $\begin{array}{l}\text { standard } \\
\text { deviation }\end{array}$ & median & $\min$ & $\max$ & total \\
\hline \multicolumn{7}{|l|}{ per test case } \\
\hline total runtime & $18 \mathrm{~m} 30 \mathrm{~s}$ & $1 \mathrm{~h} 33 \mathrm{~m}$ & $117 \mathrm{~s}$ & $162 \mathrm{~ms}$ & $15 \mathrm{~h} 21 \mathrm{~m}$ & $91 \mathrm{~h} 54 \mathrm{~m}$ \\
\hline slicing time & $28 \mathrm{~s} 150 \mathrm{~ms}$ & $41 \mathrm{~s} 808 \mathrm{~ms}$ & $14.41 \mathrm{~s}$ & $28 \mathrm{~ms}$ & $5 \mathrm{~m} 15 \mathrm{~s}$ & $2 \mathrm{~h} 19 \mathrm{~m}$ \\
\hline Yices time & $17 \mathrm{~m} 59 \mathrm{~s}$ & $1 \mathrm{~h} 33 \mathrm{~m}$ & $91.35 \mathrm{~s}$ & $110 \mathrm{~ms}$ & $15 \mathrm{~h} 20 \mathrm{~m}$ & $89 \mathrm{~h} 19 \mathrm{~m}$ \\
\hline no. of CS & $4,025.11$ & 173.76 & 4,534 & 11 & 8,609 & $1,139,600$ \\
\hline pointer operations & 8.73 & 37.74 & 8 & 4 & 242 & 2,603 \\
\hline no. of paths & 234.75 & 191.01 & 100 & 2 & 573 & 66,434 \\
\hline max. path lengths* & $2,056.21$ & $7,106.87$ & 1,031 & 122 & 32,198 & - \\
\hline total instructions ${ }^{*}$ & $1,056.20$ & 53.96 & 1,050 & 965 & 1,275 & 297,847 \\
\hline \multicolumn{7}{|l|}{ per Yices invocation } \\
\hline runtime & $267 \mathrm{~ms}$ & $4 \mathrm{~s} 986 \mathrm{~ms}$ & $21 \mathrm{~ms}$ & $1 \mathrm{~ms}$ & $5 \mathrm{~m}$ & $88 \mathrm{~h} 59 \mathrm{~m}$ \\
\hline CS size (in variables) & 891.64 & $7,706.95$ & 94 & 0 & 368,087 & - \\
\hline memory usage & $6.82 \mathrm{MB}$ & $46.54 \mathrm{MB}$ & $3.95 \mathrm{MB}$ & $3.81 \mathrm{MB}$ & $2,504.36 \mathrm{MB}$ & - \\
\hline
\end{tabular}

Path lengths are given in terms of the number of assembly instructions executed in a path. The total number of instructions per test case lists only instructions explored by SOCA, so that unreachable code is excluded. The test cases from the Verisec suite comprise between 20 and 541 LOC (average: 70 LOC; actual LOC before preprocessing and excluding header files). The minimal path length of 122 instructions correlates to 12 LOC; the maximal path length of 32,198 instructions corresponds to 503 LOC containing nested loops that are unrolled several hundred times.
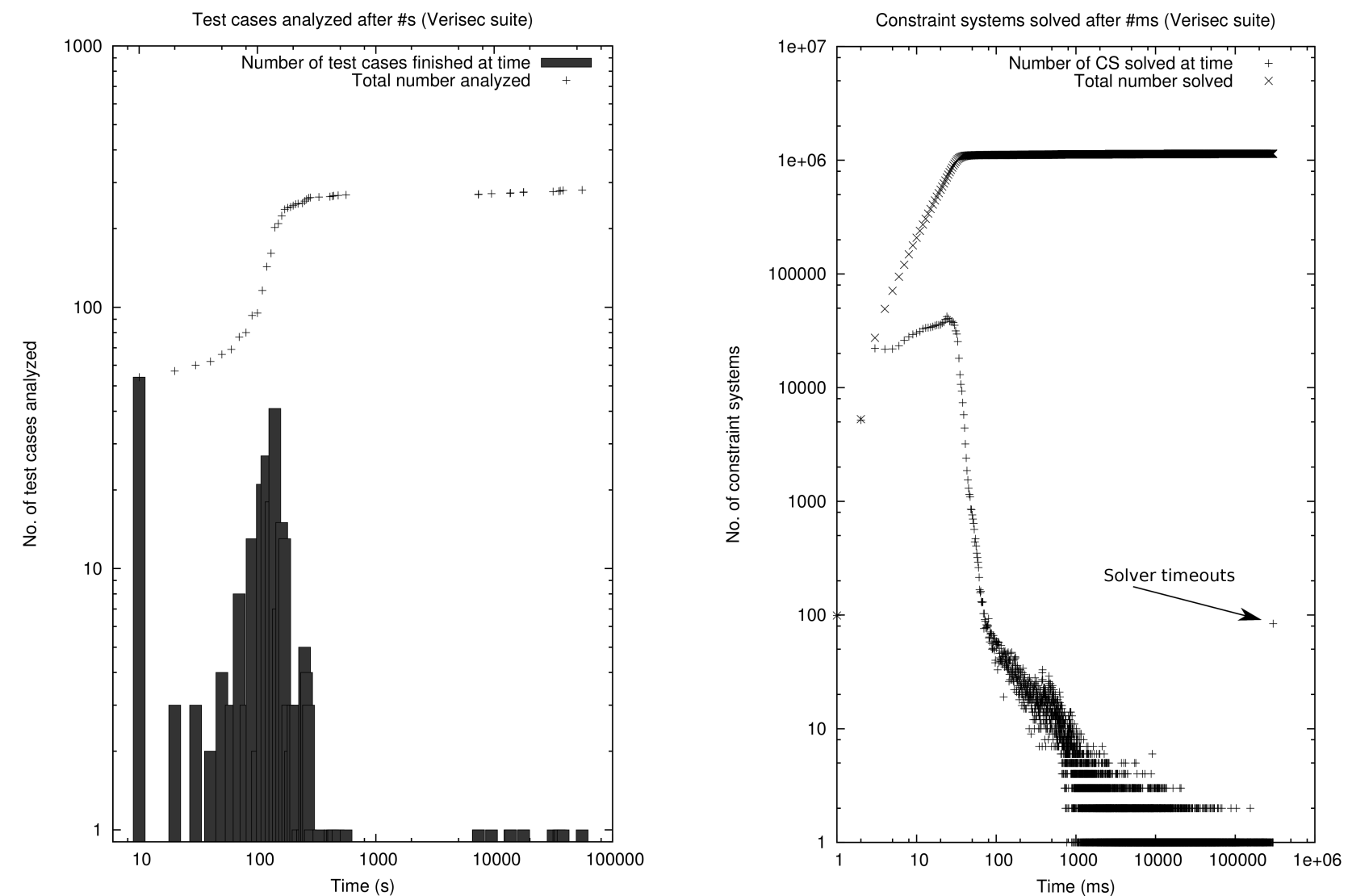

Figure 6. Performance results for the Verisec benchmark. (a) Numbers of test cases verified by time (left). (b) Numbers of constraint systems solved by time (right). 
As Table 4 testifies, the SOCA Verifier reliably detects the majority of buffer overflow errors in the benchmark, and has a competitive false-positive rate and a better discrimination rate than the other tools. Remarkable is also that the SOCA Verifier fails for only four cases of the Verisec suite: once due to memory exhaustion and three times due to missing support for certain IR instructions. Only our detection rate is lower than the one reported for LoopFrog. An explanation for this is the nature of Verisec's test cases, where static arrays are declared globally. This program setup renders Verisec easily comprehensible for source-code verification tools since the bounds of data objects are clearly identifiable in source code. In object code, however, the boundaries of data objects are not visible anymore. This makes the SOCA Verifier less effective when analyzing programs with small, statically declared buffers.

Fig. 6(a) displays details on run times; it shows the CPU times consumed by the SOCA Verifier for analyzing each test case in the Verisec benchmark. The vast majority of test cases is analyzed within three minutes per case. As can be seen in Table 5, the average computation time consumed per test case is 18.5 minutes. In total, about $92 \mathrm{CPU}$ hours were used. The memory consumption of both, the SOCA Verifier and Yices together, amounts to an average of only $140 \mathrm{MB}$ and a maximum of about $3 \mathrm{~GB}$, which is a memory capacity that is typically available in today's PCs. In addition to memory consumption we also provide the size of our constraint systems (column "CS size") in boolean variables as output by Yices. Notably, Ku reported in [33] that the SatAbs tool crashed in 73 cases and timed out in another 87 cases with a timeout of 30 minutes. The SOCA Verifier exceeds this time in only 7 cases.

Admittedly, the results reported in the literature have been obtained under a different experimental setup. In particular, a machine with only $2 \mathrm{~GB}$ of RAM is used by $\mathrm{Ku}$ in [33]. Considering that this machine, in addition to the verification tool, also executes an operating system, we assume that no more than $1.5 \mathrm{~GB}$ of RAM were available for the verifier and the underlying decision procedure. In our experiments, the SOCA Verifier together with Yices exceed a memory consumption of $1.5 \mathrm{~GB}$ in only 6 cases. These 6 cases are included in the 7 cases where the SOCA Verifier requires an analysis time above 30 minutes and can be attributed to extensive loop unwinding that results in rather long paths. The results on LoopFrog reported in [31] have been collected with runtime and memory consumption being limited to 4 hours and $4 \mathrm{~GB}$; details on the runtime behavior of LoopFrog on the Verisec benchmark are not available. Note that the processor used by Kroening et al. [31] and Ku [33], a $3 \mathrm{GHz}$ Intel Xeon, provides a processing power equal or better than the $2.3 \mathrm{GHz}$ AMD Opteron CPUs installed in our experimental setup. Thus, our data can be compared to data obtained by $\mathrm{Ku}$ and Kroening et al.; however, SatAbs is under active development and may now perform differently than in summer 2011 when our article's results were compiled.

In Fig. 6(b) we show the behavior of Yices for solving the constraint systems generated by the SOCA Verifier. For the Verisec suite, a total of 1,139,600 constraint systems were solved in 89 hours. 215,087 (19\%) of these systems express verification properties, while the others were required for computing control flows, e.g., for deciding branching conditions and resolving computed jumps. With the timeout for Yices set to 5 minutes, the solver timed out on 91 constraint systems, and $96 \%$ of the systems were solved in less than one second. Thus, the SOCA Verifier is sufficiently efficient to be used as an automated debugging tool by software developers, both regarding time efficiency and space efficiency.

Hence, despite having used a benchmark of examples that are in favor of source-code analysis, our results demonstrate that object-code analysis, as is implemented in the SOCA Verifier, can compete with stateof-the-art source-code model checkers, both qualitatively and quantitatively. However, as our tool analyzes object code, it can be employed in a much wider application domain. Unfortunately, benchmarks that include dynamic allocation and provide examples of pointer safety errors other than buffer overflows are, to the best of our knowledge, not available in the literature.

\subsection{Experiments II: Evaluating our Slicing Algorithm}

Slicing is the key for making the SOCA Verifier scale to real OS components. In this section we discuss the impact of different slicing strategies on the performance of our Verifier. We also highlight the importance of the GET \& PUT optimizations and our way of dealing with memory access in slicing, as explained in Sec. 3.2.

The experimental results we present here are, again, based on the Verisec benchmark. The SOCA Verifier is configured with the same bounds as in Sec. 5.1. As a glimpse on the overall behavior of the Verifier, we also provide data obtained from running our tool on the example program of Sec. 2.2 (compiled with gcc 4.1.2). We consider four different slicing strategies. Firstly, the Basic strategy implements "traditional" path-sensitive backwards slicing for the slicing criterion $(L, v a r)$, which means we add all statements of which var is data dependent to the slice. Memory access is handled conservatively, i.e., each load statement $l$ is assumed to be data dependent on all store statements that appear in the path before $l$. Secondly, the GETEPUT strategy combines slicing as in Basic with the GET \& PUT optimization discussed in Sec. 3.2. Our third strategy, Memory, combines Basic with our approach to handling load and store statements as explained in Sec. 3.2. Finally, we combine all three strategies in GET\&PUT + Memory.

To carry out the evaluation, we employ a modified SOCA Verifier that performs the constraint generation and the slicing four times, once for each of the above 
Table 6. Evaluation of our slicing algorithm on the running example program of Table 1

\begin{tabular}{|c|c|c|c|c|c|c|c|}
\hline \multirow[t]{2}{*}{$\begin{array}{l}\text { Slicing } \\
\text { method }\end{array}$} & \multicolumn{2}{|c|}{$\begin{array}{c}\text { CS size } \\
\text { in constraints }\end{array}$} & \multicolumn{2}{|c|}{$\begin{array}{c}\text { CS size } \\
\text { in bool vars }\end{array}$} & \multicolumn{2}{|c|}{$\begin{array}{l}\text { slice time } \\
\text { in } \mathrm{ms}\end{array}$} & \multirow[t]{2}{*}{$\begin{array}{c}\text { total runtime } \\
\text { in } \mathrm{s}\end{array}$} \\
\hline & avg. & $\max$ & avg. & $\max$. & avg. & $\max$ & \\
\hline \multicolumn{8}{|l|}{ Program from Sec. 2.2} \\
\hline Basic & 108.51 & 1,445 & 84.31 & $125^{*}$ & 5.71 & 60 & 1.21 \\
\hline GET\&PUT & 113.08 & 1,327 & 68.65 & $115^{*}$ & 6.36 & 57 & 0.87 \\
\hline Memory & 29.08 & 80 & 84.31 & $125^{*}$ & 2.68 & 6 & 0.83 \\
\hline GET\&PUT + Memory & 11.81 & 20 & 68.65 & $115^{*}$ & 2.53 & 3 & 0.56 \\
\hline \multicolumn{8}{|l|}{ Verisec benchmark } \\
\hline Basic & $9,349.15$ & 22,127 & $6,383.98$ & 378,173 & 324.16 & 5,245 & $\mathrm{n} / \mathrm{a}^{* *}$ \\
\hline GET\&PUT & $6,713.05$ & 12,907 & $6,318.72$ & 378,262 & 143.37 & 3,571 & $\mathrm{n} / \mathrm{a}^{* *}$ \\
\hline Memory & 512.42 & 20,636 & 163.22 & 12,204 & 127.67 & 3,237 & $\mathrm{n} / \mathrm{a}^{* *}$ \\
\hline GET\&PUT + Memory & 214.47 & 11,917 & 167.44 & 12,130 & 121.86 & 3,067 & $\mathrm{n} / \mathrm{a}^{* *}$ \\
\hline
\end{tabular}

The size of the constraint systems in boolean variables is reported by Yices directly. We do not know why the application of the Memory strategy increases the size of the Yices-internal representation of constraint systems in this example. Surprisingly, and importantly, the average runtime of Yices is still reduced.

** Comparing Yices' effective runtimes is not feasible since many of the constraint systems obtained with the Basic and GETESPUT strategies require several hours or even days of CPU time.

strategies. While the constraint systems generated using the strategies Basic, GETEPUT, and Memory are written to files, those generated with the GETEPUT + Memory strategy are used to progress the exploration of the program under analysis. We pass the on-disk constraint systems to Yices on a separate machine, in order to obtain their numbers of boolean variables from Yices' statistics output. We consider this to be the best means of comparing the constraint systems. Comparing Yices' runtimes is not feasible since many of the systems, especially for Basic and GETEPUT, require several hours of CPU time to solve. To give the reader an impression of the impact of different slicing strategies on the overall performance of the SOCA Verifier, we provide total runtimes of our tool for our running example program. Even for this small example program, the total runtime is halved by using full slicing, as shown in Table 6. Considering the exponential nature of SMT problems, one can estimate the high impact slicing has on our tool's performance in the Verisec benchmark or when analyzing real OS code, as is done in the next section.

To be more precise, the upper half of Table 6 contains measurements for our running example program and was obtained from 100 runs of the SOCA Verifier for each of the four slicing strategies; the lower half is dedicated to the Verisec benchmark. For each slicing method we provide the average and maximum numbers of constraints in the textual representation per constraint system, the average and maximum numbers of boolean variables per constraint system, and the average and maximum time our SOCA Verifier required for slicing and for computing the constraint systems, respectively. The table contains the aggregated data obtained from a total of about 36 million constraint systems, 9 million constraint systems per slicing strategy.
As can be seen in Table 6, the Memory strategy has the strongest impact on the number of constraints per slice and on the number of boolean variables in Yices. Our approach to slicing with respect to memory access relies on iteratively exploring the range of memory cells addressable by a particular pointer. The high impact of this strategy is because the address arguments of the majority of LD and ST statements in our example programs evaluate to constant values or very narrow address ranges. Thus, although the iterative exploration of address ranges imposes a big computational effort to the SOCA Verifier, it is vital for keeping the average sizes of constraint systems reasonably small.

Our results also show that the GETEPUT strategy, despite reducing the number of constraints per system by about $30 \%$, does not affect the numbers of boolean variables reported by Yices. Therefore, the impact of this strategy on Yices' runtime is negligible. Yet, since the strategy reduces the communication and $\mathrm{I} / \mathrm{O}$ activities of SOCA, it still contributes considerably to the scalability of our Verifier. Expectedly, we achieve the best overall performance when combining the strategies.

As can be seen, the results do not exactly match the ones presented in Sec. 5.1. Also the total number of constraint systems of about 9 million for the entire Verisec suite is different from the almost 12 million constraint systems reported earlier. The reason for this is that additional optimizations, such as extended caching strategies, were integrated into the SOCA Verifier between the two experiments. These optimizations mainly result in a more efficient implementation of the slicing algorithm and in a reduced total number of Yices invocations, thereby positively influencing the overall performance of our Verifier. 
Table 7. Performance statistics for the Linux device driver functions

\begin{tabular}{|c|c|c|c|c|c|c|}
\hline & average & $\begin{array}{l}\text { standard } \\
\text { deviation }\end{array}$ & median & $\min$ & $\max$ & total \\
\hline \multicolumn{7}{|l|}{ per test case } \\
\hline total runtime & $58 \mathrm{~m} 28 \mathrm{~s}$ & $7 \mathrm{~h} 56 \mathrm{~m}$ & $4.37 \mathrm{~s}$ & $21 \mathrm{~ms}$ & $280 \mathrm{~h} 48 \mathrm{~m}$ & $9,058 \mathrm{~h} 32 \mathrm{~m}$ \\
\hline slicing time & $8 \mathrm{~m} 35 \mathrm{~s}$ & $2 \mathrm{~h} 13 \mathrm{~m}$ & $183 \mathrm{~ms}$ & $0 \mathrm{~ms}$ & $95 \mathrm{~h} 39 \mathrm{~m}$ & $1,329 \mathrm{~h} 46 \mathrm{~m}$ \\
\hline Yices time & $48 \mathrm{~m} 36 \mathrm{~s}$ & $7 \mathrm{~h} 28 \mathrm{~m}$ & $2.55 \mathrm{~s}$ & $0 \mathrm{~ms}$ & $280 \mathrm{~h} 30 \mathrm{~m}$ & $7,531 \mathrm{~h} 51 \mathrm{~m}$ \\
\hline no. of CS & $3,591.14$ & $9,253.73$ & 369 & 0 & 53,449 & $33,383,239$ \\
\hline pointer operations & 99.53 & 312.64 & 12 & 0 & 4,436 & 925,277 \\
\hline no. of paths & 67.50 & 221.17 & 2 & 1 & 1,000 & 627,524 \\
\hline max. path lengths & 727.22 & $1,819.28$ & 97 & 1 & 22,577 & - \\
\hline \multicolumn{7}{|l|}{ per Yices invocation } \\
\hline runtime & $845 \mathrm{~ms}$ & $8 \mathrm{~s} 765 \mathrm{~ms}$ & $83 \mathrm{~ms}$ & $1 \mathrm{~ms}$ & $5 \mathrm{~m} 2 \mathrm{~s}$ & $8,295 \mathrm{~h} 56 \mathrm{~m}$ \\
\hline CS size (in variables) & $4,860.20$ & $20,256.77$ & 590 & 0 & $7,583,410$ & - \\
\hline Memory usage & $5.75 \mathrm{MB}$ & $14.76 \mathrm{MB}$ & $3.88 \mathrm{MB}$ & $3.81 \mathrm{MB}$ & $3,690.00 \mathrm{MB}$ & - \\
\hline
\end{tabular}
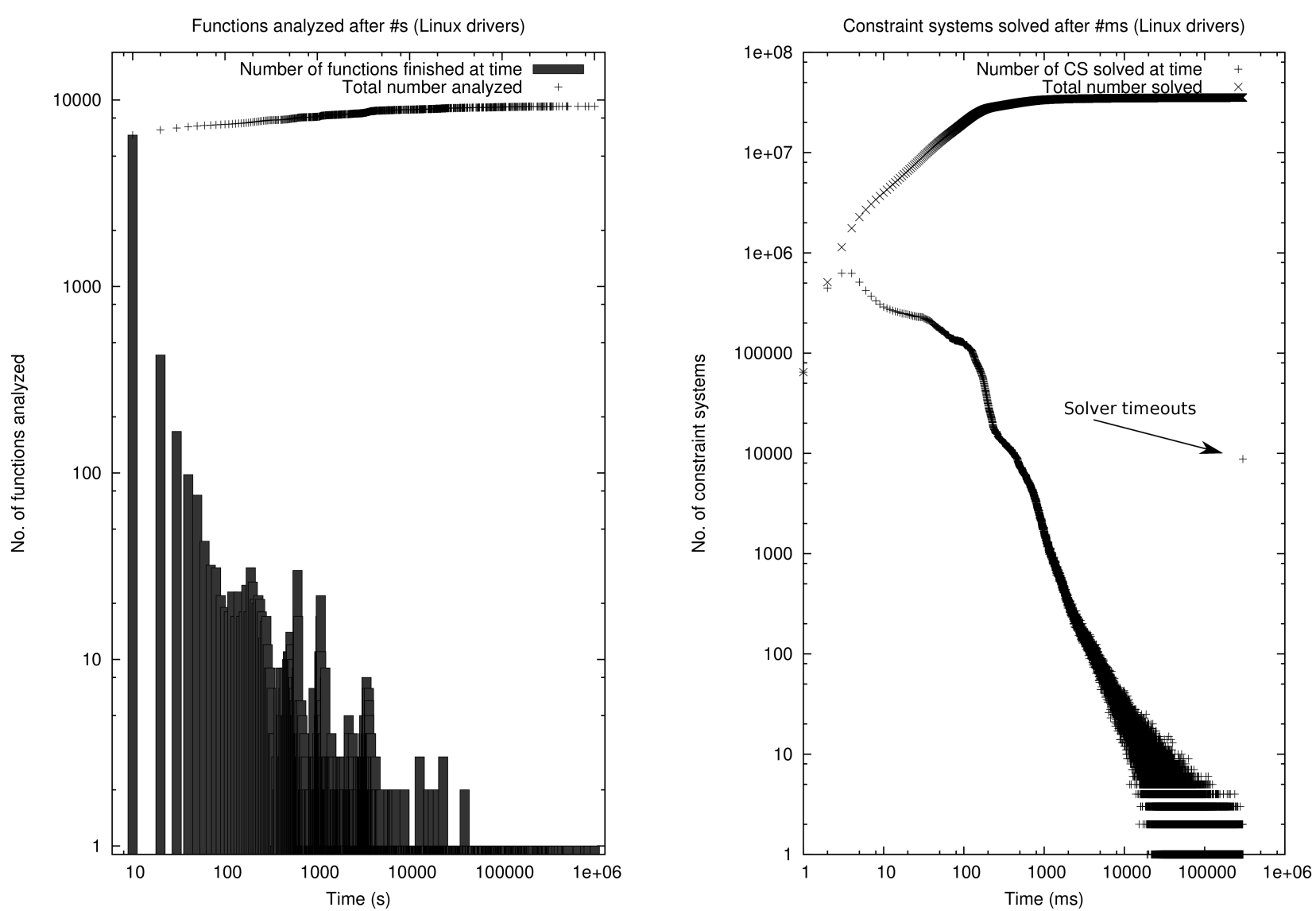

Figure 7. Performance results for the Linux device driver functions. (a) Numbers of functions verified by time (left). (b) Numbers of constraint systems solved by time (right).

\subsection{Experiments III: Linux Device Drivers}

To evaluate the scalability of the SOCA Verifier, a large set of 9,296 functions originating from 250 Linux device drivers of version 2.6.26 of the Linux kernel compiled for IA32 was analyzed by us. Our experiments employed the Linux utility $\mathrm{nm}$ to obtain a list of function symbols present in a device driver. By statically linking the driver to the Linux kernel we resolved undefined symbols in the driver, i.e., functions provided by the OS kernel that are called by the driver's functions. The SOCA technique was then applied on the resulting binary file to analyze each of the driver's functions separately. The bounds for the SOCA Verifier were set to a maximum of 1,000 paths to be analyzed, where a single instruction may appear at most 1,000 times per path, thereby effectively bound- 
ing the number of loop iterations or recursions to that depth. Moreover, Yices was configured to a timeout of 300 seconds per invocation.

Our results are summarized in Table 7 and show that $94.4 \%$ of the functions in our sample could be analyzed by the SOCA Verifier. In $67.5 \%$ of the functions, the exhaustion of execution bounds led to an early termination of the analysis. However, the analysis reached a considerate depth even in those cases, analyzing paths of lengths of up to $22,577 \mathrm{CPU}$ instructions. Interestingly, $27.8 \%$ of the functions could be analyzed exhaustively, where none of the bounds regarding the number of paths, the path lengths, or the SMT solver's timeout were reached. As depicted in Fig. 7(a), the SOCA Verifier returned a result in less than 10 minutes in the majority of cases, while the generated constraint systems were usually solved in less than $500 \mathrm{~ms}$. The timeout for Yices was hardly ever reached, as can be seen in Fig. $7(b)$.

As an aside, it must be mentioned that in $0.98 \%$ of the sample Linux driver functions (91 functions), the SOCA Verifier may have produced unsound results due to non-linear arithmetic within the generated constraint systems, which is not decidable by Yices. In addition, our Verifier failed in $5.6 \%$ of the cases (522 functions) due to either memory exhaustion, missing support for particular assembly instructions in our tool or Valgrind, or crashes of Yices.

Our evaluation shows that the SOCA Verifier scales up to real-world OS software while delivering very good performance. Being automatic and not restricted to analyzing programs available in source code only, the Verifier is an efficient tool that is capable of aiding a practitioner in debugging pointer-complex software. Further work shall add tool support for translating error traces reported by the SOCA Verifier back to source code. Doing so by hand is feasible but a huge effort, as demonstrated by us in a separate case study using the Verifier for analyzing consecutive releases of the Linux Virtual File System (VFS) implementation [39].

\section{Related Work}

There exists a wealth of related work on automated techniques for formal software verification, a survey of which can be found in [14]. We focus on more closely related work, namely on (i) model checking bytecode and assembly languages, (ii) approaches combining model checking with symbolic execution, and (iii) program slicing.

\subsection{Model Checking Bytecode \& Assembly Languages}

In recent years, several approaches to model checking compiled programs by analyzing bytecode and assembly code have been presented. In [47,52], Java PathFinder $(J P F)$ is introduced for model checking Java bytecode.
$J P F$ generates the state space of a program by monitoring a virtual machine. Model checking is then conducted on the states explored by the virtual machine, employing collapsing techniques and symmetry reduction for efficiently storing states and reducing the sizes of state spaces, respectively. These techniques are effective because of the high complexity of JPF states and the specific characteristics of the Java memory model. In contrast, the SOCA technique to verifying object code involves relatively simple states and, in difference to Java, the order of data within memory is important in IA32 object code. Similar to JPF, StEAM [35] model checks compiled $\mathrm{C}++$ programs by using a modified Internet Virtual Machine to generate a program's state space. In addition, StEAM implements heuristics to accelerate error detection.

BTOR [7] and /mc]square [42,48] are tools for model checking assembly code for micro-controllers. They accept assembly code as their input, which may either be obtained during compilation or, as suggested in [48], by disassembling a binary program. As shown in [21], the problem of disassembling a binary program is undecidable in general. Thus, the SOCA technique focuses on the verification of binary programs without the requirement of disassembling a program at once.

All tools above are explicit model checkers that require a program's entire control flow to be known in advance of the analysis. As shown in Sec. 3, this is not feasible in the presence of computed jumps. The SOCA technique is especially designed to deal with OS components that make extensive use of jump computations.

A static verifier for analyzing Windows driver programs in their binary representation is Jakstab [27]. Jakstab is similar to SOCA in that it also employs an iterative strategy for disassembling a given input program when reconstructing the program's control flow. Yet, the actual program analysis is based on abstract interpretation rather than symbolic execution, and Jakstab aims at proving the correct usage of APIs and not memory safety. To compare the performance of Jakstab and the SOCA Verifier we may again consider the Verisec suite as its programs show a close resemblance to driver binaries loaded together with a harness module. In this shallow comparison, the average runtime of Jakstab is considerably shorter (148.23s on 2,239.47 instructions per program) than that of SOCA (18m30s on 1,056.2 instructions per program, median: 117s). However, a thorough experimental comparison of the SOCA Verifier and Jak$s t a b$ is left for future work; implementing support for Windows APIs and the binary format into the SOCA Verifier is a major task and beyond the scope of this article.

\subsection{Model Checking with Symbolic Execution}

Symbolic execution was introduced by King [28] as a means of improving program testing by covering a large 
class of normal executions with a single execution, in which symbols representing arbitrary values are used as input to the program. This is exactly what our SOCA technique does, albeit not for testing but for systematic, powerful pointer safety analysis. A survey on recent trends in symbolic execution, with an emphasis on program analysis and test generation, is given in [44].

Several frameworks for integrating symbolic execution with model checking have been developed, including Symbolic JPF [43] and DART [17]. Symbolic JPF is a successor of the previously mentioned JPF. DART implements directed and automated random testing to generate test drivers and harness code to simulate a program's environment. The tool accepts $\mathrm{C}$ programs and automatically extracts function interfaces from source code. Such an interface is used to seed the analysis with a well-formed random input, which is then mutated by collecting and negating path constraints while symbolically executing the program under analysis. Unlike the SOCA Verifier, DART handles constraints on integer types only and does not support pointers and data structures.

A language agnostic tool similar to DART is $S A G E$ [18], which is used internally at Microsoft. $S A G E$ works at IA32 instruction level, tracks integer constraints as bit-vectors, and employs machine-code instrumentation in a similar fashion as we do in [39]. $S A G E$ is seeded with a well-formed program input and investigates the program space with respect to that input. Branches in the control flow are explored by negating path constraints collected during the initial execution. This differs from our approach since SOCA does not require seeding but crawls the program space automatically from a given starting point. The SOCA technique effectively computes program inputs for all paths explored during symbolic execution.

$D A R T$-like techniques, known as concolic testing, are described in $[26,49]$. These rely on performing concrete executions on random inputs while collecting path constraints along executed paths. The constraints are then used to compute new inputs that drive the program along alternative paths. In difference to this approach, SOCA uses symbolic execution to explore all paths and concretizes only for resolving computed jumps.

Related to SOCA is also work by Hansen et al. [19], which investigates the use of state-joining to speed up symbolic execution of binary programs; here, the notion of a "state" is that of a path constraint. Two states can be joined if they have identical subsequent paths, whereby the total number of paths to be analyzed is reduced. The paper also presents an implementation of state-joining, which is similar to SOCA as it is based on translating IR instructions obtained from Valgrind into constraints. Further research shall investigate whether the SOCA Verifier may be improved by incorporating the state-joining technique.

Another bounded model checker for $\mathrm{C}$ source code based on symbolic execution and SAT solving is SAT-
URN [55]. This tool is specialized on checking locking properties and null-pointer de-references and is thus not as general as SOCA. The authors of [55] show that their tool scales for analyzing the entire Linux kernel. Similar to SOCA, SATURN also employs program slicing, but in a form that is not heap-aware. Unlike the SOCA Verifier, their approach computes function summaries instead of adding the respective code to the control flow and does not handle recursion. Moreover, SOCA performs a sound analysis with respect to handling loops, interprocedural aliasing and constructs of the $\mathrm{C}$ language that are not supported by SATURN. Of course, also SOCA is only sound within the analysis bounds.

\subsection{Program Slicing}

An important SOCA ingredient other than symbolic execution is path-sensitive slicing. Program slicing was introduced by Weiser [53] as a technique for automatically selecting only those parts of a program, which may affect the values of interest computed at some location of interest. Different to conventional slicing, our slices are computed over a single path instead of an entire program, similar to what has been introduced as dynamic slicing in [29] and path slicing in [24]. In contrast to those approaches, we use conventional slicing criteria and leave a program's input initially unspecified. While collecting program dependencies is relatively easy at source code level, it becomes difficult at object code level when dependencies to the heap and stack are involved. The technique employed by SOCA for dealing with the program's heap and stack is a variation of recency abstraction [1].

\subsection{Alternative Approaches}

Alternative, recent approaches to proving pointer safety are shape analysis [54] and separation logic [46]. Most work in this area $[9,25]$ is based on analyzing the source code of a program, and calls to library functions and programming constructs such as function pointers are simply abstracted using nondeterministic assignments. A mentionable tool implementing rely-guarantee reasoning with symbolic execution for separation logic is VeriFast [23]. While VeriFast provides extensive support for pointer operations in $\mathrm{C}$, it does not facilitate automatic reasoning about programs that make use of function pointers or that contain inlined assembly code. Furthermore, VeriFast requires substantial annotations, i.e., method contracts, to be written by the software developer.

The Verifying C Compiler (VCC) [13] implements the verification of annotated $\mathrm{C}$ programs, yet, uses verification condition generation instead of symbolic execution as the underlying technology. VCC has been employed in case studies on verifying low-level OS components and 
supports inlined assembly. Similar to VeriFast, a considerate annotation overhead is imposed on the programmer. In difference to VeriFast and VCC, SOCA is designed to facilitate largely automated analysis of binary programs. Thereby, SOCA does not restrict users in the choice of programming language and does not require annotations. However, bounded techniques such as SOCA are not aiming at providing correctness proofs as VeriFast and VCC do.

Techniques applying theorem proving to verify object code and assembly code are presented in $[6,56]$. In $[6]$, the Nqthm prover is employed for reasoning about the functional correctness of implementations of well-known algorithms. The authors of [56] propose a logic-based type system for concurrent assembly code and use the Coq proof assistant for verifying programs. In contrast to our work, both theorem-proving techniques do not support "higher-order code pointers", including return pointers in function calls.

Last, but not least, validation and testing tools such as Purify [45] and Valgrind [40] must be mentioned since they have been applied successfully to identifying pointer safety problems in application software, rather than OS software. They execute an instrumented version of a given program in a protected environment in which various invalid pointer operations can be detected. However, they are meant for manual testing and do not provide means for automatically, and potentially exhaustively, exploring program behavior.

\section{Conclusions \& Future Work}

This article presented the novel SOCA technique for automatically checking pointer safety properties of pointercomplex software. Analyzing object code allows us to handle software, e.g., OS software, written in a mix of $\mathrm{C}$ and inlined assembly. Together with SOCA's symbolic execution, this simplifies pointer analysis when being confronted with function pointers, computed jumps, and pointer aliasing. SOCA achieves scalability by adopting path-sensitive slicing and the efficient SMT solver Yices. While the SOCA ingredients are well-known, the way in which we integrated these for automated object-code analysis is novel.

Much effort went into engineering our SOCA Verifier, and extensive benchmarking showed that it performs on par with state-of-the-art software model checkers and scales well when applied to Linux device driver functions. Our Verifier effectively explores semantic niches of pointer-complex software, especially OS software, which currently available model checkers and testing tools do not reach. Obviously, the lack of abstraction means that SOCA is less useful for programs that manipulate unbounded dynamic data structures, e.g., linked lists.

The application of the SOCA Verifier is, however, not restricted to verifying pointer safety. In [39] we presented a case study on retrospective verification of the Linux Virtual File System (VFS) using the SOCA Verifier for checking violations of API usage rules, such as deadlocks caused by misuse of the Linux kernel's spinlock API. The SOCA Verifier may also be ported so as to analyze binary programs for other processor architectures and operating systems. Besides enabling the use of SOCA in new application domains, a porting effort would also allow us to directly compare SOCA with tools such as DART [17] and Jakstab [27].

Future work shall be pursued along several orthogonal lines. Firstly, since device driver functions may be invoked concurrently, we plan to extend SOCA to handle concurrency by integrating dynamic partial-order reduction [16] techniques to our search strategy. To the best of our knowledge, the verification of concurrent programs with full pointer arithmetic and computed jumps is currently not supported by any automated verification tool. Secondly, we intend to evaluate different search strategies for exploring the paths of a program, employing heuristics based on, e.g., coverage criteria known from testing [57]. Thirdly, as some inputs of device drivers functions involve pointered data structures, we wish to explore whether shape analysis [9] can inform SOCA in a way that reduces the number of false positives raised. Fourthly, the SOCA Verifier shall be interfaced to the gnu debugger so that error traces can be played back in a user-friendly form, at source-code level. Finally, we are also aiming to parallelize the SOCA technique in order to benefit from currently available multi-core computer architectures. We believe that significant speedups can be achieved by modifying our algorithms so that multiple paths are explored in parallel.

Acknowledgements. We thank Bart Jacobs from KU Leuven and the anonymous reviewers of Software Tools for Technology Transfer and SPIN 2010 for their valuable comments on on this article and the previously published extended abstract, respectively, especially for pointing out some recent related work. We also thank Jim Woodcock and Daniel Kroening for their insightful remarks made at the first author's $\mathrm{PhD}$ examination. This research is partially funded by the Interuniversity Attraction Poles Programme Belgian State, Belgian Science Policy, and by the Research Fund KU Leuven.

\section{References}

1. Balakrishnan, G. and Reps, T. Recency-abstraction for heap-allocated storage. In $S A S$ '06, vol. 4134 of $L N C S$, pp. 221-239. Springer, 2006.

2. Balakrishnan, G., Reps, T., Melski, D., and Teitelbaum, T. WYSINWYX: What You See Is Not What You eXecute. In VSTTE '08, vol. 4171 of $L N C S$, pp. 202-213. Springer, 2008.

3. Ball, T., Bounimova, E., Cook, B., Levin, V., Lichtenberg, J., McGarvey, C., Ondrusek, B., Rajamani, S. K., 
and Ustuner, A. Thorough static analysis of device drivers. SIGOPS Oper. Syst. Rev., 40(4):73-85, 2006.

4. Ball, T. and Rajamani, S. K. Automatically validating temporal safety properties of interfaces. In SPIN '01, vol. 2057 of $L N C S$, pp. 103-122. Springer, 2001.

5. Barry, R. FreeRTOS: A portable, open source, mini real time kernel, 2010. http://www.freertos.org/.

6. Boyer, R. S. and Yu, Y. Automated proofs of object code for a widely used microprocessor. J. ACM, 43(1):166192, 1996.

7. Brummayer, R., Biere, A., and Lonsing, F. BTOR: Bitprecise modelling of word-level problems for model checking. In $S M T$ '08/BPR '08, pp. 33-38. ACM, 2008.

8. The C99 standard, ISO/IEC 9899:1999. Technical Report 9899:1999, International Organization for Standardization, 1999.

9. Calcagno, C., Distefano, D., O'Hearn, P., and Yang, H. Compositional shape analysis by means of bi-abduction. SIGPLAN Not., 44(1):289-300, 2009.

10. Chou, A., Yang, J., Chelf, B., Hallem, S., and Engler, D. R. An empirical study of operating system errors. In SOSP '01, pp. 73-88. ACM, 2001.

11. Clarke, E., Kroening, D., and Lerda, F. A tool for checking ANSI-C programs. In TACAS' 04 , vol. 2988 of $L N C S$, pp. 168-176. Springer, 2004.

12. Clarke, E. M., Kroening, D., Sharygina, N., and Yorav, K. SATABS: SAT-based predicate abstraction for ANSIC. In TACAS '05, vol. 3440 of $L N C S$, pp. 570-574. Springer, 2005.

13. Cohen, E., Dahlweid, M., Hillebrand, M., Leinenbach, D., Moskal, M., Santen, T., Schulte, W., and Tobies, S. VCC: A practical system for verifying concurrent C. In TPHOLs '09, vol. 5674 of LNCS, pp. 23-42. Springer, 2009.

14. D'Silva, V., Kroening, D., and Weissenbacher, G. A survey of automated techniques for formal software verification. IEEE Trans. on Computer-Aided Design of Integrated Circuits and Systems, 27(7):1165-1178, 2008.

15. Dutertre, B. and de Moura, L. The Yices SMT solver. Technical Report 01/2006, SRI, 2006. http://yices. csl.sri.com/tool-paper.pdf.

16. Flanagan, C. and Godefroid, P. Dynamic partial-order reduction for model checking software. In $P O P L$ '05, pp. 110-121. ACM, 2005.

17. Godefroid, P., Klarlund, N., and Sen, K. DART: Directed automated random testing. In $P L D I$ ' 05 , pp. 213 223. ACM, 2005.

18. Godefroid, P., Levin, M. Y., and Molnar, D. Automated whitebox fuzz testing. In NDSS '08. Internet Society (ISOC), 2008.

19. Hansen, T., Schachte, P., and Søndergaard, H. State joining and splitting for the symbolic execution of binaries. In $R V^{\prime}$ '09, vol. 5779 of $L N C S$, pp. 76-92. Springer, 2009.

20. Henzinger, T. A., Jhala, R., Majumdar, R., Necula, G. C., Sutre, G., and Weimer, W. Temporal-safety proofs for systems code. In $C A V$ '02, vol. 2402 of $L N C S$, pp. 526-538. Springer, 2002.

21. Horspool, R. N. and Marovac, N. An approach to the problem of detranslation of computer programs. Computer J., 23(3):223-229, 1980.

22. Horwitz, S., Reps, T., and Binkley, D. Interprocedural slicing using dependence graphs. ACM TOPLAS, 12(1):26-60, 1990.
23. Jacobs, B., Smans, J., and Piessens, F. A quick tour of the VeriFast program verifier. In $A P L A S$ '10, vol. 6461 of $L N C S$, pp. 304-311. Springer, 2010.

24. Jhala, R. and Majumdar, R. Path slicing. SIGPLAN Not., 40(6):38-47, 2005.

25. Josh Berdine, C. C. and O'Hearn, P. W. Symbolic execution with separation logic. In APLAS' '05, vol. 3780 of $L N C S$, pp. 52-68. Springer, 2005.

26. Kim, M. and Kim, Y. Concolic testing of the multi-sector read operation for flash memory file system. In $S B M F$ '09, vol. 5902 of $L N C S$, pp. 251-265. Springer, 2009.

27. Kinder, J. and Veith, H. Precise static analysis of untrusted driver binaries. In FMCAD '10, pp. 43-50. IEEE, 2010.

28. King, J. C. Symbolic execution and program testing. Commun. ACM, 19(7):385-394, 1976.

29. Korel, B. and Laski, J. Dynamic slicing of computer programs. J. Syst. Softw., 13(3):187-195, 1990.

30. Koshy, J. LibElf, 2009. http://wiki.freebsd.org/ LibElf.

31. Kroening, D., Sharygina, N., Tonetta, S., Tsitovich, A., and Wintersteiger, C. M. Loop summarization using abstract transformers. In ATVA '08, vol. 5311 of $L N C S$, pp. 111-125. Springer, 2008.

32. Kroening, D. and Strichman, O. Decision Procedures. Springer, 2008.

33. $\mathrm{Ku}, \mathrm{K}$. Software model-checking: Benchmarking and techniques for buffer overflow analysis. Master's thesis, U. Toronto, 2008.

34. Leung, A. and George, L. Static single assignment form for machine code. In PLDI '99, pp. 204-214. ACM, 1999.

35. Leven, P., Mehler, T., and Edelkamp, S. Directed error detection in $\mathrm{C}++$ with the assembly-level model checker StEAM. In Model Checking Software, vol. 2989 of LNCS, pp. 39-56. Springer, 2004.

36. Mühlberg, J. T. Model Checking Pointer Safety in Compiled Programs. PhD thesis, U. York, 2009. http: //etheses.whiterose.ac.uk/841/.

37. Mühlberg, J. T. and Freitas, L. Verifying FreeRTOS: from requirements to binary code. In $A V_{o} C S$ '11, vol. CSTR-1272 of Computing Science Technical Reports, Newcastle University, 2011. Short paper.

38. Mühlberg, J. T. and Lüttgen, G. BLASTing Linux code. In FMICS '06, vol. 4346 of $L N C S$, pp. 211-226. Springer, 2006.

39. Mühlberg, J. T. and Lüttgen, G. Verifying compiled file system code. In $S B M F$ '09, vol. 5902 of $L N C S$, pp. 306320 . Springer, 2009. A full version has been accepted for publication in Springer's Formal Aspects of Computing journal.

40. Nethercote, N. and Seward, J. Valgrind: A framework for heavyweight dynamic binary instrumentation. SIGPLAN Not., 42(6):89-100, 2007.

41. Nielson, F., Nielson, H. R., and Hankin, C. Principles of Program Analysis. Springer, 1999.

42. Noll, T. and Schlich, B. Delayed nondeterminism in model checking embedded systems assembly code. In Hardware and Software: Verification and Testing, vol. 4899 of LNCS, pp. 185-201. Springer, 2008.

43. Păsăreanu, C. S., Mehlitz, P. C., Bushnell, D. H., GundyBurlet, K., Lowry, M., Person, S., and Pape, M. Combining unit-level symbolic execution and system-level concrete execution for testing NASA software. In ISSTA '08, pp. 15-26. ACM, 2008. 
44. Păsăreanu, C. S. and Visser, W. A survey of new trends in symbolic execution for software testing and analysis. STTT, 11(4):339-353, 2009.

45. Rational Purify. IBM Corp., http://www.ibm.com/ software/awdtools/purify/.

46. Reynolds, J. C. Separation logic: A logic for shared mutable data structures. In LICS '02, pp. 55-74. IEEE, 2002.

47. Rungta, N., Mercer, E. G., and Visser, W. Efficient testing of concurrent programs with abstraction-guided symbolic execution. In SPIN '09, vol. 5578 of LNCS, pp. 174-191. Springer, 2009.

48. Schlich, B. and Kowalewski, S. [mc]square: A model checker for microcontroller code. In ISOLA '06, pp. 466473. IEEE, 2006.

49. Sen, K., Marinov, D., and Agha, G. CUTE: A concolic unit testing engine for C. In ESEC/FSE-13, pp. 263-272. ACM, 2005.

50. Tool Interface Standards (TIS) Committee. Executable and Linking Format (ELF) specification version 1.2, 1995. http://refspecs.freestandards.org/elf/.

51. Valgrind - Debugging and profiling Linux programs. http://valgrind.org/.

52. Visser, W., Havelund, K., Brat, G., Park, S. J., and Lerda, F. Model checking programs. FMSD, 10(2):203$232,2003$.

53. Weiser, M. Program slicing. In ICSE' '81, pp. 439-449. IEEE, 1981.

54. Wilhelm, R., Sagiv, M., and Reps, T. Shape analysis. In $C C$ '00, vol. 1781 of $L N C S$, pp. 1-16. Springer, 2000.

55. Xie, Y. and Aiken, A. SATURN: A scalable framework for error detection using boolean satisfiability. ACM TOPLAS, 29(3):16, 2007.

56. Yu, D. and Shao, Z. Verification of safety properties for concurrent assembly code. In ICFP '04, pp. 175-188. ACM, 2004.

57. Zhu, H., Hall, P. A. V., and May, J. H. R. Software unit test coverage and adequacy. ACM Comput. Surv., 29:366-427, 1997.

58. Zitser, M., Lippmann, R., and Leek, T. Testing static analysis tools using exploitable buffer overflows from open source code. SIGSOFT Softw. Eng. Notes, 29(6):97-106, 2004. 